\title{
First Order Phase Transitions in Unbounded Spin Systems. II. Completeness of the Phase Diagram
}

\author{
Christian Borgs ${ }^{1}$ and Roger Waxler ${ }^{2}$ \\ 1 Theoretische Physik, ETH-Hönggerberg, CH-8093 Zürich, Switzerland \\ 2 Dept. of Mathematics, University of British Columbia, Vancouver, BC, Canada
}

\begin{abstract}
We continue our analysis of unbounded spin systems with nearest neighbor interaction $W$ and a single spin potential $V$ which has $N$ deep and widely separated minima. In this second part we show that all translation invariant phases obeying a certain regularity condition are convex combinations of the stable phases determined in the first part of this paper. For periodic boundary conditions each stable phase contributes with the same weight in the infinite volume limit.
\end{abstract}

\section{Introduction}

In the first part [1] of this work we have shown that for an unbounded spin model with Hamiltonian

$$
H=\sum_{x} V\left(R_{x}\right)+\sum_{\langle x y\rangle} W\left(R_{x}, R_{y}\right)
$$

where $V$ has $N$ deep and widely separated minima and $W$ is a kinetic energy type interaction (see Sect. 1 of [1] for the precise assumptions), the stable phases ${ }^{1}$ are characterized by the condition that the free energy, $h_{q}^{\prime}$, of a certain truncated model is minimal. As we have seen this is enough to construct translation invariant states, $\langle\cdot\rangle_{q}$, which are small perturbations of the corresponding Gaussian approximations. Furthermore these states show exponential clustering and hence are, in the usual langauge of statistical mechanics, pure states of the sytem (1.1).

The goal of this paper is to show that these are in fact all pure states, i.e. that any translation invariant equilibrium state, $\langle\cdot\rangle$, is a convex combination of the stable states, $\langle\cdot\rangle_{q}$, constructed in [1]. To be more precise assume that $V$ and $W$ obey assumption A.0 through A.3 of [1] (guaranteeing the convergence of the cluster expansions developed in [1] and hence the existence of $\left.\langle\cdot\rangle_{q}\right)$. Assume in addition the following

\footnotetext{
${ }^{1}$ Throughout this paper we will use letters $q, q^{\prime}$, etc. to denote stable phases, while the letters $m, m^{\prime}$, etc. denote arbitrary (stable or unstable) phases
} 
Assumption R (Regularity of W). Let

$$
\alpha(R)=V(R)-\min _{R^{\prime} \in \mathbf{R}} V\left(R^{\prime}\right) .
$$

Then there is a constant $k_{1}<\infty$, possibility depending on $\beta$, such that

$$
W\left(R, R^{\prime}\right) \leqq k_{1}\left(1+\alpha(R)+\alpha\left(R^{\prime}\right)\right) .
$$

For a configuration $\underline{R}: \mathbf{Z}^{v} \rightarrow \mathbf{R}$ we set

$$
\|\underline{R}\|=\sup _{j \geqq 0} \frac{1}{|\Lambda(j)|} \sum_{x \in \Lambda(j)} \alpha\left(R_{x}\right),
$$

where $\Lambda(j)$ is the set of all points which have distance $\leqq j$ from the origin ${ }^{2}$. Generalising a little bit the notion of temperedness from $[2,4]$, we define

Definition 1.1. An equilibrium state $\langle\cdot\rangle$ is called tempered ${ }^{3}$, if $\|\underline{R}\|<\infty$ with probability one, that is, if $\left\langle\chi\left(\bigcup_{k \in \mathbf{N}}\{\underline{R} \mid\|\underline{R}\|<k\}\right)\right\rangle=1$, where $\chi(E)$ denotes the characteristic function of the event $E$.

The main result of this paper is the following theorem. We recall that $V$ depends on $N-1$ parameters $\mu=\left(\mu_{i}\right)_{i=1, \ldots, N-1}$, and that all the results of [1] were derived under the assumption that $\mu$ lies in a certain neighborhood $\mathscr{V}_{1}$ of $0 \in \mathbf{Z}^{N-1}$.

Theorem A. There is a constant $\beta^{*}<\infty$ such that all translation invariant tempered equilibrium states $\langle\cdot\rangle$ are convex combinations of the stable states $\langle\cdot\rangle_{q}$, provided $\beta \geqq \beta^{*}$ and $\mu \in \mathscr{V}_{1}$.

The proof of Theorem A is based on an idea of [5], where the corresponding theorem is shown for the Ising model at low temperatures. To explain the underlying idea consider an observable $A$ which depends only on one spin variable. Using the DLR-equations $[6,7]$ together with the translation invariance of the state $\langle\cdot\rangle$ we may rewrite the expectation value of $A$ as

$$
\langle A\rangle=\int d P\left(s_{\partial \Lambda^{c}}\right)\langle\bar{A}\rangle_{\Lambda, s_{\partial A} c},
$$

where $\Lambda$ is an arbitrary finite subvolume of $\mathbf{Z}^{v} ; \bar{A}$ denotes the average of $A$ over all translations in $\Lambda$ and $\langle\cdot\rangle_{\Lambda, s_{\partial \Lambda^{c}}}$ denotes the finite volume expectations in $\Lambda$ with boundary conditions $s_{\partial \Lambda^{c}}$. Choosing $0<\delta<1 / v$ and fixing all contours in the low temperature expansion of $\langle\bar{A}\rangle_{\Lambda, s_{\partial A^{c}}}$ which touch the boundary of $\Lambda$ (we call their union the outer contour network), one then shows that the size of this outer contour network is smaller than $\left|\partial \Lambda^{c}\right|^{1+\delta}$ with probability $1-\varepsilon(\Lambda)$, where $\varepsilon(\Lambda) \rightarrow 0$ as $\Lambda \rightarrow \mathbf{Z}^{v}$.

On the other hand, for a given outer contour network, Out, with total size smaller than $\left|\partial \Lambda^{c}\right|^{1+\delta}$, and a single term in the average $\bar{A}$, the translated observable

\footnotetext{
${ }^{2}$ We recall that we usually use the $l_{\infty}$ distance dist $(x, y)=|x-y|_{\infty}$, except for the notion of nearest neighbors, where $|x-y|=1$ denotes the $l_{1}$ distance

3 The definition of "tempered" in the sense of $[2,4]$ is obtained by substituting the norm $\|\underline{R}\|^{(2)}=\sup _{j \geqq 0}(1 /|\Lambda(j)|) \sum_{x \in \Lambda(j)} R_{x}^{2}$ for $\|\underline{R}\|$
} 
$t_{x}(A)$ is typically far away from the outer contour network. Therefore its conditional expectation value $\left\langle t_{x}(A)\right|$ Out $\rangle$ is well approximated by either $\left\langle t_{x}(A)\right\rangle_{+}=\langle A\rangle_{+}$or $\left\langle t_{x}(A)\right\rangle_{-}=\langle A\rangle_{-}$, depending on whether $t_{x}(A)$ lies in a + or in a - region of Out. Summing over Out and over the translations in $\Lambda$ one obtains the theorem by a $3 \varepsilon$ argument.

In our context two main difficulties arise when one tries to generalize this argument. The first one, the existence of unstable phases, already had to be faced in [8], where general finite range spin models with bounded, discrete spins were considered. The second is due to the fact that we are dealing with unbounded spins.

This difficulty arises at two different stages. First, the boundary conditions $R_{\partial \Lambda^{c}}$ arising in the analog of (1.4) may be arbitrarily large. This spoils the probability estimates on the existence of outer contour networks, Out, of size larger than $\left|\partial \Lambda^{c}\right|^{1+\delta}$. Secondly the conditional expectation values, $\langle\cdot \mid \mathrm{Out}\rangle$, for a fixed outer contour network, Out, still involve boundary conditions in the whole neighborhoods $\mathscr{U}_{q}$ of the minimas $R_{q}$ of $V$; while these boundary conditions are bounded for any fixed $\beta$, they are still too large ${ }^{4}$ to guarantee the convergence of the cluster expansion for $\langle\cdot\rangle_{A, R_{\partial A}}$ which we would like to use to control the difference between $\left\langle t_{x}(A)\right|$ Out $\rangle$ and $\langle A\rangle_{q}$.

We will solve these problems as follows: First we use the arguments of [2-4] to show that with probability $1-\varepsilon_{1}(\Lambda)$, where $\varepsilon_{1}(\Lambda) \rightarrow 0$ as $\Lambda \rightarrow \mathbf{Z}^{v}$, the boundary conditions appearing in the analog of (1.4) do not grow faster than $\log j$ with $j$ if $\Lambda=\Lambda(j)$. For such boundary conditions the desired probability estimates on the size of the outer contour network, Out, can be proven by a combination of the methods developed in [1] and [8]. Concerning the second problem we will in fact avoid boundary conditions growing like $\tilde{\tau}(\beta)^{1 / 2}$ in the conditional expectation values $\langle\cdot|$ Out $\rangle$ by adding a partition into small and large fields to the expansion into contours before defining the notion of an outer contour network, see Sect. 2 for details.

The organization of this paper is as follows: In Sect. 2 we recall the definition of a general equilibrium state and define the notion of an outer contour network. Assuming the validity of the probability estimates for large boundary conditions (Lemma 2.3) and those for large outer contour networks (Lemma 2.4), we well as a lemma (Lemma 2.5) which controls the difference between $\langle A\rangle_{A, R_{\partial A}}$ and $\langle A\rangle_{q}$ for boundary conditions $R_{\partial A^{c}}$ in a small neighborhood of $R_{q}$ we then prove Theorem A. Lemma 2.3 is proven in the appendix, using the methods of [2-4], and Lemma 2.4 is proven in Sect. 3. In Sect. 4 we prove Lemma 2.5 using the cluster expansion developed in [1], Sect. 7. Finally Sect. 5 is devoted to the study of periodic boundary conditions. The main result of this last section will be the following:

Theorem B. Assume that $V$ and $W$ obey the conditions A.0 through A.3 of [1]. Then, for $\mu \in \mathscr{V}_{1}$ and for $\beta$ large enough, the limit

$$
\langle\cdot\rangle_{\text {per }}=\lim _{\Lambda \rightarrow \mathbf{Z}^{v}}\langle\cdot\rangle_{\Lambda}^{\text {per }},
$$

${ }^{4}$ They grow with $\beta$ like $\tilde{\tau}(\beta)^{1 / 2}$; see Sect. 1 of [1] for the definition of $\tilde{\tau}(\beta)$ 
where $\langle\cdot\rangle_{\Lambda}^{\text {per }}$ denotes expectations in the volume $\Lambda$ with periodic boundary conditions, is well defined and is a convex combination of the stable states, $\langle\cdot\rangle_{q}$, with equal weight for each of them.

\section{The Translation Invariant Equilibrium States}

In this section we present the main steps of the proof of Theorem A. The technical details of some auxiliary estimates are deferred to Sect. 3, Sect. 4 and Appendix A. For the convenience of the reader we recall some basic notations.

We denote by $\Omega$ the set of all functions $\underline{R}: \mathbf{Z}^{v} \rightarrow \mathbf{R}$, and by $R_{\Lambda}$ the restriction of $\underline{R}$ to a bounded set $\Lambda \subset \mathbf{Z}^{v}$. A local observable is a complex valued, Borel measurable function on $\Omega$ which, for a suitable bounded set $\Lambda \subset \mathbf{Z}^{v}$, depend only on $R_{\Lambda}$. One says that $A$ is an observable in $\Lambda$, or also " $A$ has support in $\Lambda$," in this case. Finite volume expectation values with boundary conditions $R_{\partial \Lambda^{c}}$ are defined as

with

$$
\langle A\rangle_{\Lambda, R_{\partial \Lambda^{c}}}=\frac{1}{Z\left(\Lambda \mid R_{\partial \Lambda^{c}}\right)} \int A e^{-H\left(R_{\Lambda} \mid R_{\partial \Lambda^{c}}\right)} d R_{\Lambda}
$$

$$
H\left(R_{\Lambda} \mid R_{\partial \Lambda^{c}}\right)=\sum_{x \in \Lambda} V\left(R_{x}\right)+\sum_{\langle x y\rangle \cap \Lambda \neq \varnothing} W\left(R_{x}, R_{y}\right)
$$

and the obvious definition of $Z_{q}\left(\Lambda \mid R_{\partial \Lambda^{c}}\right)$. One says, a measure $d P$ on $\Omega$ obeys the DLR-equations $[6,7]$ if, for any bounded set $\Lambda$ and all bounded observables $A$ in $\Lambda^{5}$

$$
\int d P A=\int d P(\underline{R})\langle A\rangle_{\Lambda, R_{\partial A}} .
$$

A Borel measure on $\Omega$ obeying the DLR-equations is called an equilibrium measure, and the corresponding linear functional $\langle\cdot\rangle=\int \cdot d P$ is called an equilibrium state. If an equilibrium state $\langle\cdot\rangle$ fulfils the condition that $\|\underline{R}\|<\infty$ with probability 1 (see Def. 1.1 of Sect. 1) it is called tempered.

We now fix a translation invariant, tempered equilibrium state $\langle\cdot\rangle=\int \cdot d P$. Our goal is to show that it is a convex combination of the stable states $\langle\cdot\rangle_{q}$ constructed in [1]. We therefore have to show that for certain nonnegative numbers $\alpha_{q}$, where $\sum_{q} \alpha_{q}=1$, and all bounded local observables $A$,

$$
\langle A\rangle=\sum_{q} \alpha_{q}\langle A\rangle_{q}
$$

We recall that we introduced the convention that a sum or product over $q$ always denotes a sum or product, respectively, over all stable values of $q$, whereas sums or products over $m, m^{\prime}$, etc. are sums or products, respectively, over all $m \in\{1, \ldots, N\}$.

Consider a volume $\Lambda$ of the form $\Lambda=\Lambda(j)=\left\{x \in \mathbf{Z}^{v} \mid \operatorname{dist}(0, x) \leqq j\right\}$ which is a union of $L$-blocks centered in $L Z^{v}$, and a bounded observable $A$ with support in

\footnotetext{
5 In a more probabilistic language this is often formulated as: The conditional probability $P\left(d R_{\Lambda} \mid R_{A^{c}}\right)$ is given by the Gibbs measure $1 / Z\left(\Lambda \mid R_{\partial \Lambda^{c}}\right) e^{-H\left(R_{\Lambda^{\prime}} \mid R_{\left.\partial \Lambda^{c}\right)}\right.} d R_{\Lambda}$
} 
$\Lambda$. We denote by $\bar{\Lambda}$ the set of all $x \in \mathbf{Z}^{v}$ such that the translate $t_{x}(A)$ has support in $\Lambda$ and define

$$
\bar{A}=\frac{1}{|\bar{\Lambda}|} \sum_{x \in \bar{\Lambda}} t_{x}(A) .
$$

Using the translation invariance of $\langle\cdot\rangle$ (which implies that $\langle A\rangle=\langle\bar{A}\rangle$ ) together with the DLR-equations we may rewrite $\langle A\rangle$ as

$$
\langle A\rangle=\int d P(\underline{R})\langle\bar{A}\rangle_{\Lambda, R_{\partial A}} .
$$

In order to analyse the expectation values $\langle\bar{A}\rangle_{A, R_{\partial A^{c}}}$ we introduce the notation of an outer contour network. We first rewrite the partition function $Z\left(\Lambda \mid R_{\partial \Lambda^{c}}\right)$ using the same partition of unity as in [1], Sect. 2:

$$
Z\left(\Lambda \mid R_{\partial \Lambda^{c}}\right)=\sum_{\omega} \int d R_{\Lambda} \prod_{x \in \Lambda} \chi_{\omega(x)}\left(R_{x}\right) e^{-H\left(R_{\Lambda} \mid R_{\partial \Lambda^{c}}\right)},
$$

where the sum goes over all $\omega: \Lambda \rightarrow\{0,1, \ldots, N\}$ and $\chi_{\omega}(R)$ is the characteristic function of the set $\mathscr{U}_{\omega}$ (we recall that $\mathscr{U}_{1}, \ldots, \mathscr{U}_{N}$ are certain neighborhoods of the minima, $R_{1}, \ldots, R_{N}$, of the potential $V$, with growing diameter $2 \tau(\beta)^{1 / 2}$ as the inverse temperature $\beta \rightarrow \infty$, and that $\left.\mathscr{U}_{0}=\mathbf{R} \backslash \bigcup_{m=1}^{N} \mathscr{U}_{m}\right)$.

Differing a little bit from the notation introduced in [1] we define: a block $\square\left(x^{\prime}\right)$ in $\Lambda$ is $m$-correct, if $\square\left(x^{\prime}\right)$ does not touch $\partial \Lambda^{c}$ and if $\omega(x)=m \forall x$ with $\operatorname{dist}\left(x, \square\left(x^{\prime}\right)\right) \leqq 1$. If $\square\left(x^{\prime}\right)$ is $q$-correct for some stable $q$ we say that $\square\left(x^{\prime}\right)$ is a stable book. As before the union of all incorrect blocks is denoted $B(\omega)$.

Let $\chi_{\text {small }}^{(m)}\left(R_{\square\left(x^{\prime}\right)}\right)$ be the characteristic function of the event that $\left|R_{x}-R_{m}\right| \leqq B \forall x \in \square\left(x^{\prime}\right)$, with some constant $B$ to be chosen later. We define

$$
\begin{aligned}
& \chi_{\text {small }}\left(R_{\square\left(x^{\prime}\right)}\right)=\sum_{m=1}^{N} \chi_{\text {small }}^{(m)}\left(R_{\square\left(x^{\prime}\right)}\right), \\
& \chi_{\text {large }}\left(R_{\square\left(x^{\prime}\right)}\right)=1-\chi_{\text {small }}\left(R_{\square\left(x^{\prime}\right)}\right),
\end{aligned}
$$

and indroduce the notation

$$
\chi_{\text {small }}\left(R_{X}\right)=\prod_{\square\left(x^{\prime}\right) \subset X} \chi_{\text {small }}\left(R_{\square\left(x^{\prime}\right)}\right)
$$

for a union, $X$, of $L$-blocks in $\Lambda$, and similarly for $\chi_{\text {large }}\left(R_{X}\right)$. Introducing the above partition of unity for all stable blocks we rewrite $Z\left(\Lambda \mid R_{\partial \Lambda}\right)$ as

$$
Z\left(\Lambda \mid R_{\partial \Lambda^{c}}\right)=\sum_{\omega, X} \int d R_{\Lambda} \chi_{\text {large }}\left(R_{X}\right) \chi_{\text {small }}\left(R_{X^{c}}\right) \prod_{x \in \Lambda} \chi_{\omega(x)}\left(R_{x}\right) e^{-H\left(R_{\Lambda} \mid R_{\partial \Lambda^{c}}\right)},
$$

where the sum goes over pairs $(\omega, X)$ consisting of a function $\omega: \Lambda \rightarrow\{0, \ldots, N\}$ and a union of stable blocks (stable with respect to $\omega$ ). Given $X$ and $\omega, X^{c}$ denotes the union of all stable blocks which are not in $X$.

We now define the outer contour network of a configuration $(\omega, X)$. We consider the union, $\operatorname{Irr}=X \cup B(\omega)$, and define: The connected component, $E$, of Irr which 
touches $\partial \Lambda^{c}$ is called the boundary component of Irr. A component, $C$, of Irr is called an $m$-component of Irr if $C$ does not touch $\partial \Lambda^{c}$ and if $\omega=m$ on $\partial_{e} C$. A $m$-component, $C$, of $\operatorname{Irr}$ is called large, if $a_{m} \operatorname{diam} C>\tau / 3$ (with $a_{m}$ as defined in [1], Sect. 3), and small otherwise. Finally, a component, $C$, of Irr is called a canonical component of Irr, if $C$ is large and if there is no small component, $C^{\prime}$, of Irr for which $C$ lies in the interior of $C^{\prime}$. We define the outer contour network of the configuration $(\omega, X)$ as the triple $\underline{\text { Out }}=\left(\right.$ Out, $\omega_{\text {Out }}, X($ Out $\left.)\right)$, where Out is the union of the boundary component with all canonical components of Irr, $\omega_{\text {Out }}$ is the restriction of $\omega$ to Out and $X(\mathrm{Out})=X \cap$ Out; for $m=1, \ldots, N ; V_{m}$ denotes the union of all components, $C$, of $\Lambda \backslash$ Out for which $\omega=m$ on $\partial C$.

Resumming all $(\omega, X)$ which lead to the same outer contour network we rewrite

$$
\begin{aligned}
Z\left(\Lambda \mid R_{\partial \Lambda^{c}}\right)= & \sum_{\text {Out }} \int d R_{\text {Out }} \chi_{\text {large }}\left(R_{X(\mathrm{Out})}\right) \prod_{x \in \mathrm{Out}} \chi_{\omega(x)}\left(R_{x}\right) e^{-H\left(R_{\mathrm{Out}} \mid R_{\partial \Lambda^{c}}\right)} \\
& \cdot \prod_{m}^{\prime} Z_{m}^{\text {small }}\left(V_{m} \mid R_{\partial V_{m}^{c}}\right) \prod_{q} \tilde{Z}_{q}\left(V_{q} \mid R_{\partial V_{q}^{c}}\right)
\end{aligned}
$$

where the product $\prod^{\prime}$ runs over all unstable $m$, and the product over $q$ runs over all stable $q . Z_{m}^{\text {small }}$ is the partition function obtained from $Z_{m}^{\text {dil }}$ by restricting the sum over $\omega$ in the definition of $Z_{m}^{\text {dil }}$ (see Sect. 2 of [1]) to a sum over all configurations containing only small external contours, and $\tilde{Z}_{q}\left(V_{q} \mid \cdot\right)$ is obtained from $Z_{q}^{\text {dil }}\left(V_{q} \mid \cdot\right)$ by inserting an additional characteristic function $\chi_{\text {small }}\left(R_{\square\left(x^{\prime}\right)}\right)$ for each block touching $\partial V_{q}$.

Remark 2.1. We recall that $a_{q}=0$ for stable $q$; therefore all canonical components of Irr must be $m$-components for some unstable $m$. It follows that the volumes $V_{q}$ in (2.9) are always volumes without holes, while $V_{m}$ may have holes if $m$ is unstable.

We define approximations $\alpha_{q}(\Lambda)$ to $\alpha_{q}$ by

with

$$
\alpha_{q}(\Lambda)=\int d P(\underline{R}) \sum_{\underline{\text { Out }}} P\left(\text { Out } \mid R_{\partial \Lambda^{c}}\right) \alpha_{q}(\underline{\text { Out }})
$$

and

$$
\alpha_{q}(\underline{\text { Out }})=\frac{1}{\sum_{q^{\prime}}\left|V_{q^{\prime}}\right|} \times\left|V_{q}\right|
$$

$$
\begin{aligned}
P\left(\underline{\text { Out }} \mid R_{\partial \Lambda^{c}}\right)= & \frac{1}{Z\left(\Lambda \mid R_{\partial \Lambda^{c}}\right)} \int d R_{\text {Out }} \chi_{\text {large }}\left(R_{X(\mathrm{Out})}\right) \prod_{x \in \mathrm{Out}} \chi_{\omega(x)}\left(R_{x}\right) \\
& \cdot e^{-H\left(R_{\mathrm{Out}} \mid R_{\partial \Lambda^{c}}\right)} \prod_{m}^{\prime} Z_{m}^{\text {small }}\left(V_{m} \mid R_{\partial V_{m}^{c}}\right) \prod_{q} \tilde{Z}_{q}^{\mathrm{dil}}\left(V_{q} \mid R_{\partial V_{m}^{c}}\right) .
\end{aligned}
$$

We want to show that the limits $\alpha_{q}=\lim _{\Lambda \rightarrow \mathbf{z}^{v}} \mathrm{a}_{q}(\Lambda)$ exist and satisfy Eq. (2.4) for all bounded local observables $A$. Both statements, and hence Theorem A, are an immediate corollary of the following Proposition 2.1. We denote by $\Lambda \nearrow \mathbf{Z}^{v}$ a sequence of volumes $\Lambda(j), j \nearrow \infty$, which are unions of $L$-cubes. $\mathscr{V}_{1} \subset \mathbf{R}^{N-1}$ is the region defined in Lemma 3.1 of [1]. 
Proposition 2.1. There is a constant $\beta^{*}<\infty$ such that, for $\mu \in \mathscr{V}_{1}$ and $\beta \geqq \beta^{*}$, and a suitable choice of $L$ and $B$,

$$
\left|\langle A\rangle-\sum_{q} \alpha_{q}(\Lambda)\langle A\rangle_{q}\right| \rightarrow 0
$$

as $\Lambda \nearrow \mathbf{Z}^{v}$, for all bounded, local observables, $A$.

Corollary 2.2. For $\mu, \beta, B$ and $L$ as in Proposition 2.1, the limits

$$
\lim _{\Lambda \rtimes \mathbf{Z}^{v}} \alpha_{q}(\Lambda)=\alpha_{q}
$$

exist, and fullfill Eq. (2.4) for all bounded local observables $A$.

Proof of Corollary 2.2. We only have to prove (2.14). Consider the observables $A_{q}=\chi_{q}\left(R_{x}\right)$, where $x$ is some fixed point in $\mathbf{Z}^{v}$ (e.g. the origin) and $q$ is stable. For $\beta$ large,

$$
\left\langle A_{q}\right\rangle_{q^{\prime}}=M_{q q^{\prime}}=\delta_{q q^{\prime}}+\delta M_{q q^{\prime}},
$$

where $\delta M_{q q^{\prime}} \rightarrow 0$ as $\beta \rightarrow \infty$. Therefore $M$ is invertible for $\beta$ large enough, and $M^{-1}$ is bounded. Using (2.13) it follows that

$$
\sum_{q^{\prime}}\left(M^{-1}\right)_{q q^{\prime}}\left\langle\chi_{q^{\prime}}\left(R_{x}\right)\right\rangle-\alpha_{q}(\Lambda)
$$

goes to zero as $\Lambda \nearrow \mathbf{Z}^{v}$. This proves (2.14) and hence the corollary.

We are now going to prove the proposition. The proof is based on three technical lemmas which will be proved in separate sections. For a volume $\Lambda$ without holes (that is, a volume $\Lambda$ such that $\Lambda^{c}$ is connected) we denote

$$
\langle A\rangle_{\Lambda, R_{\partial \Lambda^{c}}}^{q}=\frac{1}{Z_{q}\left(\Lambda \mid R_{\partial \Lambda^{c}}\right)} \int A \prod_{x \in \partial \Lambda} \chi_{q}\left(R_{x}\right) e^{-H\left(R_{\Lambda} \mid R_{\left.\partial \Lambda^{c}\right)}\right.} d R_{\Lambda}
$$

with the obvious definition of $Z_{q}\left(\Lambda \mid R_{\partial \Lambda^{c}}\right)$.

Lemma 2.3. There is a constant $a<\infty$, and a function $\varepsilon_{1}(j) \rightarrow 0$ as $j \rightarrow \infty$, such that

$$
\alpha\left(R_{x}\right) \leqq a \log j \quad \forall x \in \partial \Lambda(j)^{c}
$$

with probability $1-\varepsilon_{1}(j)$, provided the equilibrium measure $P$ is tempered.

Lemma 2.4. Denote by $\sum^{\prime \prime}$ the sum over all outer contour networks such that

$$
\left|\Lambda \backslash \bigcup_{q} V_{q}\right|>\left|\partial \Lambda^{c}\right|^{1+\delta}
$$

Assume that $R_{\partial \Lambda^{c}}$ is chosen in such a way that $\alpha\left(R_{x}\right) \leqq a \log j$ for all $x \in \partial \Lambda^{c}=\partial \Lambda(j)^{c}$, and let $L \geqq L_{0}$, where $L_{0}$ is the constant from Theorem 3.3 of [1]. Then there are constants $\beta_{1}=\beta_{1}(L)$ and $B_{1}=B_{1}(L)$ such that

$$
\sum_{\text {Out }}^{\prime \prime} P\left(\underline{\text { Out }} \mid R_{\partial \Lambda^{c}}\right) \leqq \varepsilon_{2}(\Lambda),
$$

provided $\beta \geqq \beta_{1}, \mu \in \mathscr{V}_{1}$ and $B \geqq B_{1} . \varepsilon_{2}(\Lambda)$ is a constant going to zero as $\Lambda \nearrow \mathbf{Z}^{v}$. 
Lemma 2.5. There are constants $L_{0}<\infty$ and $\beta_{2}=\beta_{2}(L, B)$ such that, for $B<\infty$, $L \geqq L_{0}, \mu \in \mathscr{V}_{1}, \beta \geqq \beta_{2}(L, B)$ and all volumes $\Lambda$ which are unions of $L$-blocks centered in $L Z^{v}$

$$
\left|\langle A\rangle_{\Lambda, R_{\partial \Lambda^{c}}}^{q}-\langle A\rangle_{q}\right| \leqq\|A\| K^{|\operatorname{supp} A|} e^{-M \operatorname{dist}(A, \partial \Lambda)},
$$

provided $q$ is stable and $\left|R_{x}-R_{q}\right| \leqq B \forall x \in \partial \Lambda^{c} . M>0$ and $K<\infty$ are constants not depending on $A$ or $\Lambda$.

Proof of Proposition 2.1. The proof relies on two facts:

i) outer contour networks for which $\left|\Lambda \backslash \bigcup_{q} V_{q}\right|$ is larger than $\left|\partial \Lambda^{c}\right|^{1+\delta}$ are suppressed as $\Lambda \nearrow \mathbf{Z}^{v}$ (Lemma 2.4);

ii) for a given outer contour network with $\left|\Lambda \backslash \bigcup_{q} V_{q}\right| \leqq\left|\partial \Lambda^{c}\right|^{1+\delta}$ most terms $t_{x}(A)$ in the average $\bar{A}$ lie deep inside a region $V_{q}$; therefore their conditional expectation values $\left\langle t_{x}(A) \mid \underline{\text { Out }}\right\rangle_{\Lambda, R_{\partial \Lambda^{c}}}$ are well approximatized by $\left\langle t_{x}(A)\right\rangle_{q}=\langle A\rangle_{q}($ Lemma 2.5).

Summing over Out and integrating over the boundary conditions $R_{\partial \Lambda^{c}}$ we will obtain the proposition by a $4 \varepsilon$-argument.

We fix $L \geqq L_{0}$ according to Lemmas 2.4 and 2.5 and choose $B \geqq B_{1}(L), \beta \geqq$ $\max \left\{\beta_{1}(L), \beta_{2}(L, B)\right\}$. We fix $a$ according to Lemma 2.3 and $\delta<1 / v$. Given $\varepsilon$ and a bounded local observable $A$, we will show that the left-hand side of (2.13) can be made smaller than $4 \varepsilon$ by choosing $\Lambda$ large enough.

Given $A$ and $\varepsilon$, we choose $d_{0}$ so large that

$$
\|A\| K^{|\operatorname{supp} A|} e^{-M d_{0}} \leqq \varepsilon .
$$

For an outer contour network Out with $\left|\Lambda \backslash \bigcup_{q} V_{q}\right| \leqq\left|\partial \Lambda^{c}\right|^{1+\delta}$, let $D_{q}$ denote the union of all cubes, $\square\left(x^{\prime}\right)$, in $V_{q}$ which touch the outer contour network, and set $\widetilde{V}_{q}=V_{q} \backslash D_{q}$. By the definition of an outer contour network, all fields $R_{x}$ with $x \in D_{q}$ obey the condition $\left|R_{x}-R_{q}\right| \leqq B$. Therefore the conditional expectation value $\left\langle t_{x}(A) \mid \underline{\text { Out }}\right\rangle_{\Lambda, R_{\partial \Lambda^{c}}}$ can be rewritten as

$$
\int d \tilde{P}\left(R_{\partial \tilde{V}_{q}^{c}}\right)\left\langle t_{x}(A)\right\rangle_{\tilde{V}_{q}, R_{\partial} \tilde{V}_{q}^{c}},
$$

provided $t_{x}(A)$ has support in $\tilde{V}_{q} ; d \tilde{P}$ is a probability measure with support in the set of all fields $R_{\partial \tilde{V}_{q}^{c}}$ which obey the condition $\left|R_{x}-R_{q}\right| \leqq B \forall x \in \partial \tilde{V}_{q}^{c}$. Applying Lemma 2.5 we obtain that

$$
\left|\left\langle t_{x}(A) \mid \underline{\text { Out }}\right\rangle_{\Lambda, R_{\partial \Lambda^{c}}}-\langle A\rangle_{q}\right| \leqq \varepsilon,
$$

provided $x \in \tilde{\Lambda}_{q}$, where $\tilde{\Lambda}_{q}$ is defined as the set of all $x \in \mathbf{Z}^{v}$ such that $t_{x}(A)$ has support in $\tilde{V}_{q}$ and $\operatorname{dist}\left(t_{x}(A), \partial \tilde{V}_{q}\right) \geqq d_{0}$. On the other hand,

$$
\left|\frac{\left|\tilde{\Lambda}_{q}\right|}{|\bar{\Lambda}|}-\alpha_{q}(\underline{\text { Out }})\right|=\left|\frac{\left|\tilde{\Lambda}_{q}\right|}{|\bar{\Lambda}|}-\frac{\left|V_{q}\right|}{\sum_{q^{\prime}}\left|V_{q^{\prime}}\right|}\right| \leqq O\left(\frac{\left(L+d_{0}\right)\left|\partial \Lambda^{c}\right|^{1+\delta}}{|\Lambda|}\right)
$$

provided $\mid$ Out $\left.|\leqq| \partial \Lambda^{c}\right|^{1+\delta}$. Using the fact that $\left|\left\langle t_{x}(A) \mid \underline{\text { Out }}\right\rangle_{\Lambda, R_{\partial \Lambda^{c}}}\right| \leqq\|A\|$ to bound 
the terms for which $x \notin \bigcup_{q} \tilde{\Lambda}_{q}$ we obtain that

$$
\left|\langle\bar{A} \mid \underline{\text { Out }}\rangle_{\Lambda, R_{\partial \Lambda^{c}}}-\sum_{q} \alpha_{q}(\underline{\text { Out }})\langle A\rangle_{q}\right| \leqq 2 \varepsilon,
$$

provided $\Lambda=\Lambda(j)$ is large enough and $\left|\Lambda \backslash \bigcup_{q} V_{q}\right| \leqq\left|\partial \Lambda^{c}\right|^{1+\delta}$. We finally use the identity

$$
\langle A\rangle=\int d P(\underline{R})\langle\bar{A}\rangle_{\Lambda, R_{\partial \Lambda^{c}}}=\int d P(\underline{R}) \sum_{\text {Out }} P\left(\underline{\text { Out }} \mid R_{\partial \Lambda^{c}}\right)\langle\bar{A} \mid \underline{\text { Out }}\rangle_{\Lambda, R_{\partial \Lambda^{c}}}
$$

together with Lemma 2.4 and Lemma 2.5 to conclude that

$$
\left|\langle A\rangle-\sum_{q} \alpha_{q}(\Lambda)\langle A\rangle_{q}\right| \leqq 4 \varepsilon
$$

provided $\Lambda=\Lambda(j)$ is chosen so large that $2\|A\| \varepsilon_{2}(\Lambda) \leqq \varepsilon$ and $2\|A\| \varepsilon_{1}(j) \leqq \varepsilon$. This completes the proof of Proposition 2.1.

\section{The Suppression of Unstable Phases}

In this section we prove Lemma 2.4. We first bound $P\left(\underline{\text { Out }} \mid R_{\partial \Lambda^{c}}\right)$ for a given outer contour network; this will reduce us to an ordinary contour model with hard core interaction. The sum $\sum$ " over Out can then be estimated by the methods of [8]. Throughout this section we will assume that $\mu \in \mathscr{V}_{1}$ with $\mathscr{V}_{1}$ as defined in Lemma 3.1 of [1].

We start with a lower bound on $Z\left(\Lambda \mid R_{\partial \Lambda^{c}}\right)$. We choose a stable value of $q$ and use the fact that $1 \geqq \chi_{q}$ to bound, for $\Lambda=\Lambda(j)$,

$$
Z\left(\Lambda \mid R_{\partial \Lambda^{c}}\right) \geqq Z_{q}^{\mathrm{dil}}(\Lambda) e^{-k_{1}\left(1+a \log j+k_{2}\right)\left|\partial \Lambda^{c}\right|},
$$

where $k_{1}$ is the constant from Assumption $\mathrm{R}$ (see Sect. 1) and $k_{2}=\max _{R \in \mathscr{U}_{q}} \alpha(R)$. Choosing $L \geqq L_{0}$ and $\beta \geqq \beta_{2}(L)$ as in Theorem 3.3 of [1] we may bound $Z_{q}^{\text {dil }}(\Lambda)$ from below by $e^{-h_{q}^{\prime}|\Lambda|-C_{1}\left|\partial \Lambda^{c}\right|}=e^{-h|\Lambda|-C_{1}\left|\partial \Lambda^{c}\right|}$.

On the other hand $Z_{m}^{\text {small }}\left(V_{m} \mid R_{\partial V_{m}^{c}}\right)$ and $\tilde{Z}_{q}^{\text {dil }}\left(V_{q} \mid R_{\partial V_{q}^{c}}\right)$ may be bounded by $Z_{m}^{\prime}\left(V_{m}\right)$ and $Z_{q}^{\text {dil }}\left(V_{q}\right)=Z_{q}^{\prime}\left(V_{q}\right)$, respectively ${ }^{6}$. We use Lemma 3.2 of [1] together with the bound $\sum_{m}^{\prime}\left|\partial V_{m}^{c}\right|+\sum_{q}\left|\partial V_{q}^{c}\right| \leqq \mid$ Out $\mid$ to estimate the product of these partition functions. Inserting the resulting bounds into the definition (2.12) of $P\left(\underline{\text { Out }} \mid R_{\partial \Lambda^{c}}\right)$ we obtain

$P\left(\underline{\text { Out }} \mid R_{\partial \Lambda^{c}}\right) \leqq e^{\left(k_{1}+a k_{1} \log j+k_{1} k_{2}+C_{1}\right)\left|\partial \Lambda^{c}\right|} e^{\left(h+C_{1}\right)|\mathrm{Out}|}$

$$
\cdot e^{-\sum_{m} a_{m}\left|V_{m}\right|} \int d R_{\text {Out }} \chi_{\text {large }}\left(R_{X(\mathrm{Out})}\right) \prod_{x \in \mathrm{Out}} \chi_{\omega(x)}\left(R_{x}\right) e^{-H\left(R_{\mathrm{Out}}\right)}
$$

\footnotetext{
${ }^{6}$ We recall that the truncated partition functions $Z_{m}^{\prime}(\Lambda)$ were obtained from $Z_{m}^{\text {dil }}(\Lambda)$ by truncating the activities of the unstable contours, see [1], Sect 3, Definition 31
} 
To bound the integral in (3.1) we use the fact that all blocks $\square\left(x^{\prime}\right)$ in Out which do not touch $\partial \Lambda^{c}$ are either incorrect or contain a large field. If $\square\left(x^{\prime}\right)$ contains a large field, $\omega(x)$ takes a constant value $q$ on $\square\left(x^{\prime}\right)$ which is stable. We therefore may bound $H\left(R_{\square\left(x^{\prime}\right)}\right)$ by

$$
\sum_{x \in \square\left(x^{\prime}\right)}\left[e_{q}+\frac{\eta}{2}\left(R-R_{q}\right)^{2}\right] .
$$

by our assumption A.1, [1]. Using the definition of $\chi_{\text {large }}\left(R_{\square\left(x^{\prime}\right)}\right)$ and bounding the integrals

$$
\int_{|R| \geqq B} e^{-(\eta / 2) R^{2}} d R \leqq e^{-(\eta / 2) B^{2}} \int_{-\infty}^{+\infty} e^{-(\eta / 2) R^{2}} d R,
$$

one easily finds the estimate

$$
\int d R_{\square\left(x^{\prime}\right)} \chi_{\omega(x)}\left(R_{x}\right) \chi_{\text {large }}\left(R_{\square\left(x^{\prime}\right)}\right) e^{-H\left(R_{\square\left(x^{\prime}\right)}\right)} \leqq e^{-(\eta / 2) B^{2}}\left(2(2 \pi / \eta)^{1 / 2} e^{-e_{0}}\right)^{\left|\square\left(x^{\prime}\right)\right|}
$$

with $e_{0}=\min _{q} e_{q}$. Continuing as in the proof of Lemma 3.1 of [1] to bound the remaining integrals we obtain the bound

with

$$
\int d R_{\text {Out }} \chi_{\text {large }}\left(R_{X(\text { Out })}\right) \prod_{x \in \text { Out }} \chi_{\omega(x)}\left(R_{x}\right) e^{-H\left(R_{\text {Out }}\right)} \leqq e^{k_{3}\left|\partial \Lambda^{c}\right|-\left(\tau^{\prime}+e_{0}\right) \mid \text { Out } \mid}
$$

$$
e^{-\tau^{\prime}}=\max \left\{e^{-\tau}, 2(2 \pi / \eta)^{1 / 2} e^{-(\eta / 2) B^{2} L^{-d}}\right\}
$$

and $k_{3}=L\left(\tau^{\prime}+\log C_{0}\right) . \tau$ and $C_{0}$ are the constants from Lemma 3.1 of [1]; the factor $k_{3}\left|\partial \Lambda^{c}\right|$ arises because we defined the outer contour network in such a way that it contains the blocks $\square\left(x^{\prime}\right)$ touching $\partial \Lambda^{c}$, even if none of these blocks contributes a small factor. Using Lemma 3.2 of [1] a second time to bound $h \leqq e_{0}+C_{1}$ we finally obtain the estimate

$$
P\left(\underline{\text { Out }} \mid R_{\partial \Lambda^{c}}\right) \leqq e^{\left(K+a k_{1} \log j\right)\left|\partial \Lambda^{c}\right|} e^{-\left(\tau^{\prime}-2 C_{1}\right)|\mathrm{Out}|} e^{-\sum_{m} a_{m}\left|V_{m}\right|},
$$

with $K=C_{1}+k_{1}\left(1+k_{2}\right)+k_{3}$.

We are now ready to bound the sum $\sum^{\prime \prime}$ over Out. We extract a factor

$$
e^{-(\kappa / 2)\left|\partial \Lambda^{c}\right|^{1+\rho}} \geqq e^{-\sum_{m}\left(a_{m} / 2\right)\left|V_{m}\right|} e^{-\left(\tau^{\prime} / 2\right)|\mathrm{Out}|},
$$

with $\kappa=\min \left\{\tau^{\prime}, \min \left\{a_{m}: a_{m} \neq 0\right\}\right\}$, resum all outer contour networks which differ only by $X$ (Out), and finally relax the condition (2.16) in the sum $\sum^{\prime \prime}$. We obtain the bound

$$
\begin{aligned}
\sum_{\text {Out }}^{\prime \prime} P\left(\underline{\text { Out }} \mid R_{\partial \Lambda^{c}}\right) \leqq & e^{\left(K+a k_{1} \log j-(\kappa / 2)\left|\partial \Lambda^{c}\right|^{\rho}\right)\left|\partial \Lambda^{c}\right|} \\
& \cdot \sum_{\left(\text {Out }, \omega_{\text {Out }}\right)}^{\prime} e^{-\sum_{m}\left(a_{m} / 2\right)\left|V_{m}\right|} e^{-\left(\tau^{\prime} / 2-2 C_{1}-\log 2\right) \mid \text { Out } \mid},
\end{aligned}
$$

where the sum $\sum^{\prime}$ goes over all sets Out $\subset \Lambda$ which are unions of $L$-blocks and over all functions $\omega_{\text {Out }}$ : Out $\rightarrow\{0,1, \ldots, N\}$ such that 
i) Out contains all blocks $\square\left(x^{\prime}\right)$ in $\Lambda$ which touch $\partial \Lambda^{c}$,

ii) $\omega_{\text {Out }}$ takes a constant value $m \neq 0$ on $\partial C^{c}$ for each component $C$ of $\Lambda \backslash$ Out and

iii) all components $C$ of Out which do not touch $\partial \Lambda^{c}$ obey the condition $\operatorname{diam} C>\tau / 3 a_{m}$ if $\omega=m$ on $\partial_{e} C$.

The sets $V_{m}, m=1, \ldots, N$, are defined as before, see Sect. 2 .

Lemma 2.4 now follows immediately from (3.4), the observation that $\tau^{\prime}$ can be made arbitrarily large by choosing $\beta$ and $B$ large, and the following

Lemma 3.1. There is a constant $\tau_{0}$, depending only on $N$ and $v$, such that

$$
\sum_{\left(\text {Out }, \omega_{\text {Out }}\right)}^{\prime} e^{-\sum_{m}\left(a_{m} / 2\right)\left|V_{m}\right|} e^{-\tilde{\tau}|\mathrm{Out}|} \leqq e^{\mid \partial \Lambda^{c}} \mid,
$$

provided $\tau \geqq \tilde{\tau} \geqq \tau_{0}$.

Remark 3.1. Lemma 3.1 can actually be proven by the methods of [8], Sect. 2.3 to Sect. 3.3. We give an alternative proof below.

Proof. We introduce an auxiliary contour model as follows: A pair $\gamma=\left(\operatorname{supp} \gamma, \omega_{\gamma}\right)$ where supp $\gamma$ is a connected union of $L$-blocks centered in $L Z^{v}$ and $\omega_{\gamma}$ is a function from supp $\gamma$ into $\{0,1, \ldots, N\}$ is called a contour, if $\omega_{\gamma}$ takes a constant value $m \neq 0$ on $\partial C^{c}$ for each connected component of $\mathbf{Z}^{v} \backslash \operatorname{supp} \gamma$. The sets Ext $\gamma$ and $\operatorname{Int}_{m} \gamma$, $m=1, \ldots, N$, are defined as usual as the infinite component of $\mathbf{Z}^{\nu} \backslash \operatorname{supp} \gamma$ and as the union of those components $C$ for which $\omega_{\gamma}=m$ on $\partial C^{c}$, respectively. $\gamma$ is called an $m$-contour if $\omega_{\gamma}=m$ on $\partial(\operatorname{Ext} \gamma)^{c}$.

The activity of an $m$-contour $\gamma$ is defined as

$$
z(\gamma)= \begin{cases}0 & \text { if } \operatorname{diam} \gamma \leqq \tau / 3 a_{m} \\ e^{-\tilde{\tau}|\operatorname{supp} \gamma|} & \text { if } \operatorname{diam} \gamma>\tau / 3 a_{m}\end{cases}
$$

and the partition function, $\mathscr{Z}_{m}^{\text {dil }}$, is defined as

$$
\mathscr{Z}_{m}^{\mathrm{dil}}(\Lambda)=\sum_{\left\{\gamma_{1}, \ldots, \gamma_{n}\right\}} \prod_{m^{\prime}=1}^{N} e^{-\left(a_{m^{\prime}} / 2\right)\left|V_{m^{\prime}}\right|} \prod_{i=1}^{n} z\left(\gamma_{i}\right) .
$$

As usual the sum goes over sets of pairwise compatible contours $\gamma_{1}=$ $\left(\operatorname{supp} \gamma_{1}, \omega_{1}\right), \ldots, \gamma_{n}=\left(\operatorname{supp} \gamma_{n}, \omega_{n}\right)$ such that $\omega$ can be extended to a function on $\Lambda$ which is constant on $C \cup \partial C^{c}$ for all connected components $C$ of $\Lambda \backslash \cup \operatorname{supp} \gamma_{i}$, and which takes the value $m$ on $\partial \Lambda$. $V_{m^{\prime}}$ denotes the union of all those components $C$ for which $\omega=m^{\prime}$ on $\partial C$. We only allow "dilute configurations," $\gamma_{1}, \ldots, \gamma_{n}$, i.e. we require that $\operatorname{dist}\left(\Lambda^{c}, \operatorname{supp} \gamma_{i} \cup \operatorname{Int} \gamma_{i}\right)>L$ for all $i=1, \ldots, n$. Note that $z(\gamma)=0$ for all stable contours $\gamma$; therefore $\mathscr{Z}_{q}^{\text {dil }}(\Lambda)=1$ if $a_{q}=0$.

With the help of these definitions we rewrite the left-hand side of (3.5) as

$$
\sum_{\left(E, \omega_{E}\right)} e^{-\tilde{\tau}|E|} \prod_{m} \mathscr{Z}_{m}^{\mathrm{di1}}\left(\operatorname{Int}_{m} E\right)
$$

where the sum goes over all boundary contours $\left(E, \omega_{E}\right)$, that is over all connected unions, $E$, of $L$-blocks in $\Lambda$ and all functions, $\omega_{E}$, from $E$ into $\{0,1, \ldots, N\}$ such that $E$ contains all blocks touching $\partial \Lambda^{c}$ and such that $\omega_{E}$ is constant on $\partial C^{c}$ for 
each component $C$ of $\Lambda \backslash E$. We will bound the partition functions, $\mathscr{Z}_{m}^{\mathrm{dil}}\left(\operatorname{Int}_{m} E\right)$, in (3.7) by the analog of Theorem 3.3 ([1], Sect. 3) for $\mathscr{Z}_{m}^{\text {dil }}$.

As usual (see, for example, [8], Sect. 1 or [9], Sect. 2) one may rewrite $\mathscr{Z}_{m}^{\mathrm{dil}}(\Lambda)$ as a sum over pairwise compatible $m$-contours $\gamma_{1}^{m}, \ldots, \gamma_{n}^{m}$ :

with

$$
\mathscr{Z}_{m}^{\mathrm{dil}}(\Lambda)=e^{-\left(a_{m} / 2\right)|\Lambda|} \sum_{\left\{\gamma_{1}^{m}, \ldots, \gamma_{n}^{m}\right\}} \prod_{i=1}^{n} \rho\left(\gamma_{i}^{m}\right)
$$

$$
\rho\left(\gamma^{m}\right)=z\left(\gamma^{m}\right) e^{-\left(a_{m} / 2\right) \mid \text { supp } \gamma^{m} \mid} \prod_{m^{\prime}} \frac{\mathscr{Z}_{m^{\prime}}^{\mathrm{dil}}\left(\operatorname{Int}_{m^{\prime}} \gamma^{m}\right)}{\mathscr{Z}_{m}^{\mathrm{dil}_{m}}\left(\operatorname{Int}_{m^{\prime}} \gamma^{m}\right)} .
$$

We also introduce a truncated partition function, $\mathscr{Z}_{m}^{\prime}(\Lambda)$, by replacing $\rho(\gamma)$ by

$$
\rho^{\prime}(\gamma)=\min \left\{\rho(\gamma), e^{-(\tilde{\tau} / 6)|\operatorname{supp} \gamma|}\right\} .
$$

For $\tilde{\tau}$ large enough, depending only on $v$ and $N, \mathscr{Z}_{m}^{\prime}(\Lambda)$ can be analysed by a convergent cluster expansion. One obtains that

$$
\left|\mathscr{Z}_{m}^{\prime}(\Lambda)+f_{m}^{\prime}\right| \Lambda|| \leqq O\left(e^{-(\tilde{\tau} / 6)}\right)\left|\partial \Lambda^{c}\right|,
$$

where $f_{m}^{\prime}$ is the free energy of the truncated model. Since all contours contributing to $f_{m}^{\prime}$ are larger than $\tau / 3 a_{m}$, we may bound

$$
\left|f_{m}^{\prime}-\frac{a_{m}}{2}\right| \leqq e^{-(\tilde{\tau} / 12) \times\left(\tau / 3 a_{m}\right)} \leqq \frac{a_{m}}{36 \tilde{\tau}^{2}} \leqq \frac{a_{m}}{2},
$$

provided $\tilde{\tau}$ is large enough and $\tau \geqq \tilde{\tau}$. We conclude that $f^{\prime}=\min f_{m}^{\prime} \geqq 0$. Using the analog of Theorem 3.3 of [1] (see also Theorem 1.7 of [8]) this shows that

$$
\mathscr{Z}_{m}^{\text {dil }}\left(\operatorname{Int}_{m} E\right) \leqq e^{\left|\partial\left(\operatorname{Int}_{m} E\right)^{c}\right|} \leqq e^{|E|}
$$

provided $\tau \geqq \tilde{\tau} \geqq \tau_{0}$, where $\tau_{0}$ is a constant depending only on $N$ and $v$. Inserting this bound into (3.7) and summing over $\left(E, \omega_{E}\right)$ one immediately obtains the lemma.

\section{Proof of Lemma 2.5}

In this section we prove Lemma 2.5 by a suitable generalization of the cluster expansion for $\langle A\rangle_{q, \Lambda}^{\text {dil }}$ derived in Sect. 7 of [1]. We first derive a cluster expansion for

$$
Z_{q}\left(\Lambda \mid R_{\partial \Lambda^{c}}\right)=\int d R_{\Lambda} \prod_{x \in \partial \Lambda} \chi_{q}\left(R_{x}\right) e^{-H\left(R_{\Lambda} \mid R_{\left.\partial \Lambda^{c}\right)}\right.}
$$

Starting from the identity

$$
\prod_{x \in \partial \Lambda} \chi_{q}\left(R_{x}\right)=\sum_{\omega: \Lambda \rightarrow\{0, \ldots, N\}}^{\prime} \prod_{x \in \Lambda} \chi_{\omega(x)}\left(R_{x}\right),
$$

where the sum $\sum^{\prime}$ goes over all $\omega$ such that $\omega(x)=q$ for $x \in \partial \Lambda$, we proceed as in [1] to obtain a contour expansion for $Z_{q}\left(\Lambda \mid R_{\partial \Lambda^{c}}\right)$,

$$
Z_{q}\left(\Lambda \mid R_{\partial \Lambda^{c}}\right)=\sum_{\mathscr{C}} \int \chi_{q}\left(R_{\Lambda \backslash \mathscr{C}}\right) e^{-H\left(R_{\Lambda} \backslash \mathscr{E} \mid R_{\left.\partial \Lambda^{c}\right)}\right.} \prod_{\gamma \in \mathscr{C}} e^{-\psi_{q}\left(\gamma \mid R_{\left.\partial_{e} \gamma\right)}\right)} d R_{\Lambda \backslash \mathscr{C}}
$$


The sum $\sum_{\mathscr{C}}$ goes over sets of pairwise compatible $q$-contours $\gamma$ with supp $\gamma \subset \Lambda$. $\psi_{q}$ is defined as before (Eq. (2.11) of [1]) and obeys a bound ${ }^{7}$

$$
e^{-\psi_{q}\left(\gamma \mid R_{\left.\partial_{e} \gamma\right)}\right.} \leqq e^{-\left(e_{q}+(\tau / 3)\right)|\operatorname{supp} \gamma|}
$$

Recall that $\Lambda \backslash \mathscr{C}$ stands for $\Lambda \backslash \bigcup_{\gamma \in \mathscr{C}} \operatorname{supp} \gamma$ and that $\partial_{e} \gamma$ was defined as $\partial(V(\gamma))^{c}$ (Eq. (2.7) of [1]). Note that (4.3) differs from the expansion for $Z_{q}^{\text {dil }}(\Lambda)$ in [1] by the dependence of $e^{-H}$ on $R_{\partial \Lambda^{c}}$ and the fact that the set $\mathscr{C}$ in (4.3) may contain contours $\gamma$ which touch $\partial \Lambda^{c}$.

We continue as in [1] with an expansion of the Gibbs factor $e^{-H\left(R_{\Lambda \backslash \xi \mid} \mid R_{\partial \Lambda^{c}}\right)}$ around the corresponding Gaussian approximation, with the difference that we absorb the perturbing interaction, $W_{q}\left(\phi_{x}, \phi_{y}\right)$, into the definition of the perturbing potential if $\langle x y\rangle$ intersects $\partial \Lambda^{c}$. More precisely we define (with, as before, $\left.\phi_{x}=R_{x}-R_{0}\right)$

$$
\begin{aligned}
\tilde{W}_{q}\left(\phi, \phi^{\prime}\right) & =W\left(R_{q}+\phi, R_{q}+\phi^{\prime}\right)-\frac{\kappa_{q}}{2}\left(\phi-\phi^{\prime}\right)^{2}, \\
\tilde{V}_{q}\left(\phi_{x}\right) & =V\left(R_{q}+\phi_{x}\right)-e_{q}-\frac{m_{q}^{2}}{2} \phi_{x}^{2}+\sum_{\substack{y \in \partial \Lambda^{c} \\
|x-y|=1}} W_{q}\left(\phi_{x}, \phi_{y}\right),
\end{aligned}
$$

and $^{8}$, for $X \subset \Lambda \backslash \mathscr{C}$,

$$
\tilde{F}_{X}\left(\phi_{X}\right)=\sum_{\substack{X_{1}, B \\ X=X 1 \cup X(B)}} \prod_{x \in X_{1}}\left\{\chi_{q}\left(\phi_{x}+R_{q}\right) e^{-\tilde{V}_{q}\left(\phi_{x}\right)}-1\right\} \prod_{\langle x y\rangle \in B}\left\{e^{-\tilde{W}_{q}\left(\phi_{x}, \phi_{y}\right)}-1\right\},
$$

where $B$ denotes a set of nearest neighbor pairs and $X(B)$ denotes the corresponding set of points. We obtain the expansion

$$
\begin{aligned}
& Z_{q}\left(\Lambda \mid R_{\partial \Lambda^{c}}\right)=e^{-e_{q}|\Lambda|} Z_{q}^{(0)}\left(\Lambda \mid R_{\partial \Lambda^{c}}\right) \sum_{\mathscr{C} X \subset \Lambda \backslash \mathscr{C}} \sum_{X} \int d \phi_{X(\mathscr{G})} e^{-H_{q}^{(0)}\left(R_{X(\mathscr{G})} \mid R_{\partial \Lambda^{c}}\right)} \\
& \cdot \tilde{F}_{X}\left(\phi_{X}\right) \frac{Z_{q}^{(0)}\left(\Lambda_{1} \mid \phi_{\partial X(\mathscr{C}) \cup \partial \Lambda^{c}}\right)}{Z_{q}^{(0)}\left(\Lambda \mid \phi_{\partial \Lambda^{c}}\right)} \prod_{\gamma \in \mathscr{C}} e^{e_{q}|\operatorname{supp} \gamma|-\psi_{q}\left(\gamma \mid R_{\partial_{e} \gamma}\right)},
\end{aligned}
$$

where $X(\mathscr{C})=X \cup\left(\Lambda \cap \partial_{e} \mathscr{C}\right)$ and $\Lambda_{1}=\Lambda \backslash(X(\mathscr{C}) \cup \operatorname{supp} \mathscr{C})$. $H_{q}^{(0)}$ and $Z_{q}^{(0)}$ are the quadratic and Gaussian approximations to $H$ and $Z$, respectively, in $\mathscr{U}_{q}$ (see Eq. (4.3) and (4.6) of [1] for the definitions).

The above expansion is an expansion into "excitations" $(\mathscr{C}, X)$, coupled through a ratio of Gaussian partition functions. As in [1] we decouple the connected components of these excitations with an expansion for this ratio (see [1], Sect. 4, Eq. (4.8)). We obtain the polymer representation

$$
Z_{q}\left(\Lambda \mid R_{\partial \Lambda^{c}}\right)=e^{-e_{q}|\Lambda|} Z_{q}^{(0)}\left(\Lambda \mid R_{\partial \Lambda^{c}}\right) \sum_{\mathbb{P}} \prod_{P \in \mathbb{P}} \rho\left(P \mid R_{\partial \Lambda^{c}}\right),
$$

\footnotetext{
${ }^{7}$ Throughout this section we assume that $q$ is stable

${ }^{8}$ With a slight abuse of notation we suppress the dependence of $\tilde{V}_{q}\left(\phi_{x}\right)$ and $\tilde{F}_{X}\left(\phi_{X}\right)$ on $R_{\partial \Lambda^{c}}$
} 
where the sum goes over sets $\mathbb{P}=\left\{P_{1}, P_{2}, \ldots\right\}$ of polymers in $\Lambda$ (i.e. connected unions of $L$-blocks in $\Lambda$ ) such that $P_{i}$ and $P_{j}$ have no face in common for $i \neq j$. $\rho\left(P \mid R_{\partial \Lambda^{c}}\right)$ is defined as

$$
\begin{aligned}
\rho\left(P \mid R_{\partial \Lambda^{c}}\right)= & \sum_{\mathscr{C}, X, \Gamma} \frac{1}{|\Gamma| !} \frac{1}{Z_{q}^{(0)}\left(\bar{X} \cup \operatorname{supp} \mathscr{C} \mid \phi_{\partial \Lambda^{c}}\right)} \int d \phi_{\bar{X}} e^{-H_{q}^{(0)}\left(\phi_{\bar{X}} \mid \phi_{\partial \Lambda^{c}}\right)} \\
& \cdot \tilde{F}_{X}\left(\phi_{X}\right) \prod_{\gamma \in \mathscr{C}} e^{e_{q}|\operatorname{supp} \gamma|-\psi_{q}\left(\gamma \mid R_{\partial_{e} \gamma}\right)} \prod_{\Gamma \in \Gamma} \tilde{W}\left(\Gamma \mid \phi_{\partial X(\mathscr{C}) \cup \partial \Lambda^{c}}\right)
\end{aligned}
$$

where the sum goes over all triples $(\mathscr{C}, X, \Gamma)$ such that supp $\mathscr{C} \cup \bar{X} \cup X(\Gamma)=P$, and

$$
\tilde{W}\left(\Gamma \mid \phi_{\partial X(\mathscr{C}) \cup \partial \Lambda^{c}}\right)=\int d s_{\Gamma} \partial^{\Gamma}\left\{\log Z_{q}^{(0)}\left(\Lambda_{1}, s_{\Gamma} \mid \phi_{\partial X(\mathscr{C}) \cup \partial \Lambda^{c}}\right)-\log Z_{q}^{(0)}\left(\Lambda, s_{\Gamma} \mid \phi_{\partial \Lambda^{c}}\right)\right\} .
$$

As in [1] $\Gamma$ denotes a set of faces separating $L$-blocks in $\Lambda, \Gamma$ denotes a sequence $\left(\Gamma_{1}, \ldots, \Gamma_{|\Gamma|}\right), X(\Gamma)$ is the set of $L$-blocks which have at least one face in common with $\Gamma$ and $X(\Gamma)=\bigcup_{\Gamma \in \Gamma} X(\Gamma)$. The $s$-dependent partition functions $Z_{q}^{(0)}\left(\cdot, s_{\Gamma} \mid \cdot\right)$ are defined by interpolation in the Hamiltonian $H_{q}^{(0)}$, and $\bar{X}$ is the smallest union of $L$-blocks in $\Lambda \backslash \operatorname{supp} \mathscr{C}$ such that $X(\mathscr{C}) \subset \bar{X}$ and $\operatorname{dist}(\Lambda \backslash(\bar{X} \cup \operatorname{supp} \mathscr{C}), X(\mathscr{C}))<r_{0}$, where $r_{0}$ is a constant to be chosen below.

Note that for polymers $P$ not touching $\partial \Lambda$ the activities $\rho\left(P \mid R_{\partial \Lambda^{c}}\right)$ do not depend $^{9}$ on $R_{\partial \Lambda^{c}}$ and are equal to the activities $\rho(P)$ obtained in [1] for the partition function $Z_{q}^{\text {dil }}(\Lambda)$. The following proposition immediately gives convergence of the Mayer expansion for $\log Z_{q}\left(\Lambda \mid \phi_{\partial \Lambda^{c}}\right)$.

Proposition 4.1. Given $b<\infty$ there is a choice of $L_{0}<\infty$, such that, for all $L \geqq L_{0}$ and all $B<\infty$ one can choose $r_{0}=r_{0}(L), \beta_{1}=\beta_{1}(L, B), \tau_{1}=\tau_{1}(L, B)$ in such a way that

$$
\left|\rho\left(P \mid R_{\partial \Lambda^{c}}\right)\right| \leqq e^{-b|P|},
$$

provided $\left|\phi_{x}\right| \leqq B$ for all $x \in \partial \Lambda^{c}$ and $\beta \geqq \beta_{1}, \tau=\tau(\beta, L) \geqq \tau_{1}$.

We first prove the following lemma, which is the analog of Lemma 5.1 of [1]; $\eta$ and $\eta^{\prime \prime}$ are the constants from Assumption A.1.

Lemma 4.2. For all $c<\infty$ there is a constant $L_{0}=L_{0}\left(c, \eta, \eta^{\prime \prime}, v\right)$ such that, for $L \geqq L_{0}, \varepsilon>0$ and a suitable choice of $r_{0}$ (depending on $\varepsilon, L, \eta, \eta^{\prime \prime}$ and $v$ )

$$
\begin{gathered}
\sum_{\substack{\Gamma \text { s.t. } \\
\operatorname{supp} \mathscr{C} \cup \bar{X} \cup X(\Gamma)=P}} \frac{1}{|\Gamma| !} \prod_{\Gamma \in \Gamma}\left|\tilde{W}\left(\Gamma \mid \phi_{\partial X(\mathscr{C}) \cup \partial \Lambda^{c}}\right)\right| \leqq \exp \left\{\frac{\varepsilon}{2} \sum_{x \in \partial X(\mathscr{C})} \phi_{x}^{2}\right\} \\
\cdot \exp \left\{f_{1}\left(1+B^{2}\right)|\bar{X} \cup \operatorname{supp} \mathscr{C}|-c L^{-v}|P \backslash(\bar{X} \cup \operatorname{supp} \mathscr{C})|\right\},
\end{gathered}
$$

provided $\left|\phi_{x}\right| \leqq B$ for all $x \in \partial \Lambda^{c} . f_{1}$ is a constant depending only on $L, v, \eta$ and $\eta^{\prime \prime}$. Proof. We closely follow the proof of Proposition 5.3 and Lemma 5.1 in [1]. For a

\footnotetext{
9 There is no dependence of $\rho\left(P \mid R_{\partial \Lambda^{c}}\right)$ on $R_{\partial \Lambda^{c}}$ via $\tilde{W}(\Gamma \mid \cdot)$ for these polymers, because the contributions from $\Lambda \backslash P$ in (4.8b) cancel if $P$ does not touch $\partial \Lambda$
} 
given $\Gamma$ let $\tilde{C}^{(1)}$ and $\tilde{C}^{(2)}$ be the covariances corresponding to $Z_{q}^{(0)}\left(Y_{1}, s_{\Gamma} \mid \phi_{\partial X(\mathscr{G}) \cup \partial \Lambda^{c}}\right)$ and $Z_{q}^{(0)}\left(Y_{2}, s_{\Gamma} \mid \phi_{\partial \Lambda_{c}}\right)$, respectively, with $Y_{2}=\bar{X} \cup \operatorname{supp} \mathscr{C} \cup X(\Gamma)$ and $Y_{1}=Y_{2} \backslash$ $(X(\mathscr{C}) \cup \operatorname{supp} \mathscr{C})$. Using the fact that the contributions from $\Lambda \backslash Y_{2}$ cancel in (4.8b) we rewrite

$$
\tilde{W}\left(\Gamma \mid \phi_{\partial X(\mathscr{G}) \cup \partial \Lambda^{c}}\right)=\tilde{W}(\Gamma)+\frac{1}{2} \sum_{x, y \in \partial X(\mathscr{G}) \cup \partial \Lambda^{c}} \phi_{x} B_{x y}^{(1)} \phi_{y}-\frac{1}{2} \sum_{x, y \in \partial \Lambda^{c}} \phi_{x} B_{x y}^{(2)} \phi_{y},
$$

where $\tilde{W}(\Gamma)$ is obtained from $W(\Gamma)$ as defined in [1] by substituting $\tilde{C}^{(i)}$ for $C^{(i)}$; $B_{x y}^{(i)}$ is defined as

$$
B_{x y}^{(i)}=B_{x y}^{(i)}(\Gamma)=\sum_{\substack{u, v \in Y_{i} \\|u-x|=|v-y|=1}} \int d s_{\Gamma} \partial^{\Gamma} \tilde{C}_{u v}^{(i)}
$$

Using the inequality $\phi_{x}^{2}+\phi_{y}^{2} \geqq 2\left|\phi_{x} \phi_{y}\right|$ and the symmetry $B_{x y}^{(i)}=B_{y x}^{(i)}$ we bound

$$
\begin{aligned}
& \sum_{\Gamma:|\Gamma|=n}\left|\tilde{W}\left(\Gamma \mid \phi_{\partial X(\mathscr{C}) \cup \partial \Lambda^{c}}\right)\right| \\
& \leqq \sum_{\Gamma:|\Gamma|=n}|\tilde{W}(\Gamma)|+\frac{1}{2} \sum_{x \in \partial X(\mathscr{G})} \phi_{x}^{2} \sum_{\substack{y \in \mathbf{Z}^{v} \\
\Gamma:|\Gamma|=n}}\left|B_{x y}^{(1)}(\Gamma)\right| \\
& \quad+\frac{1}{2} \sum_{x \in \partial \Lambda^{c}} \phi_{x}^{2} \sum_{\substack{y \in \mathbf{Z}^{v} \\
\Gamma:|\Gamma|=n}}\left|B_{x y}^{(1)}(\Gamma)\right|+\frac{1}{2} \sum_{\substack{x \in \partial \Lambda^{c} \\
n_{x}}} \phi_{x}^{2} \sum_{\substack{y \in \mathbf{Z}^{v} \\
\Gamma:|\Gamma|=n}}\left|B_{x y}^{(2)}(\Gamma)\right| .
\end{aligned}
$$

We now extract a factor $e^{-m_{0} \text { ro/2 }}$ from the sum over $y$ in the second term on the right-hand side and use the fact that only those $x$ contribute to the last two terms for which $x \in \partial \Lambda^{c} \cap\left(Y_{2} \cup \partial Y_{2}^{c}\right) \subset \partial Y_{2}^{c}$, because $B_{x y}^{(i)}=0$ if $\operatorname{dist}\left(x, Y_{2}\right) \geqq 2$. Continuing as in the proof of Proposition 5.3 of [1] we obtain the estimate

$$
\begin{aligned}
\sum_{\Gamma:|\Gamma|=n}\left|\tilde{W}\left(\Gamma \mid \phi_{\partial X(\mathscr{G}) \cup \partial \Lambda^{c}}\right)\right| \leqq & d_{1}^{n+1} e^{-n\left(m_{0} L / 4 v\right)}\left\{\frac{e^{m_{0} L / 2}}{L}|\bar{X} \cup \operatorname{supp} \mathscr{C}|\right. \\
& \left.+\frac{e^{-\left(m_{0} / 2\right)\left(r_{0}-L\right)}}{2} \sum_{x \in \partial X(\mathscr{C})} \phi_{x}^{2}+e^{m_{0} L / 2} B^{2}\left|\partial Y_{2}^{c}\right|\right\},
\end{aligned}
$$

where $d_{1}$ is the constant from Proposition 5.3 of [1] (it depends only on $v, \eta$ and $\left.\eta^{\prime}\right)$. We now bound $\left|\partial Y_{2}^{c}\right| \leqq\left(3^{\nu} / L\right)\left|Y_{2}\right|$ and use the fact that $|X(\Gamma)| \leqq 2 L^{d}|\Gamma| \leqq$ $2|\Gamma||\bar{X} \cup \operatorname{supp} \mathscr{C}|$ for all terms which contribute to the above sum (if $\bar{X} \cup \operatorname{supp} \mathscr{C}=\varnothing, \quad Y_{2}=Y_{1}$ and $\tilde{W}\left(\Gamma \mid \phi_{\partial X(\mathscr{C}) \cup \partial \Lambda^{c}}\right)=0 ; \quad$ if $\bar{X} \cup \operatorname{supp} \mathscr{C} \neq \varnothing$, the inequality is trivial); we therefore may bound

$$
\left|\partial Y_{2}^{c}\right| \leqq \frac{3^{v}}{L}(1+2|\Gamma|)|\bar{X} \cup \operatorname{supp} \mathscr{C}| \leqq \frac{3^{v+n}}{L}|\bar{X} \cup \operatorname{supp} \mathscr{C}|,
$$

which shows that

$$
\begin{aligned}
\left|\tilde{W}\left(\Gamma \mid \phi_{\partial X(\mathscr{G}) \cup \partial \Lambda^{c}}\right)\right| \leqq & \left(3 d_{1} e^{-\left(m_{0} L / 4 v\right)}\right)^{n}\left\{\frac{d_{1} e^{m_{0} L / 2}}{L} 3^{v}\left(1+B^{2}\right)|\bar{X} \cup \operatorname{supp} \mathscr{C}|\right. \\
& \left.+\frac{d_{1} e^{-\left(m_{0} / 2\right)\left(r_{0}-L\right)}}{2} \sum_{x \in \partial X(\mathscr{G})} \phi_{x}^{2}\right\} .
\end{aligned}
$$


Given $c<\infty$ we choose $L_{0}$ so large that

$$
3 d_{1} e^{-\left(m_{0} L / 4 v\right)} \leqq \frac{1}{2} e^{-2 c}
$$

and $r_{0}=r_{0}\left(\varepsilon, L, \eta, \eta^{\prime \prime}, v\right)$ so large that

$$
d_{1} e^{-\left(m_{0} / 2\right)\left(r_{0}-L\right)} \leqq \varepsilon .
$$

Lemma 4.2 now follows from the bound (4.9) in the same way as Lemma 5.1 follows from Proposition 5.3 in [1].

Proof of Proposition 4.1. Using Lemma 4.2 together with the bound (4.4) we are left with a bound on a sum over terms of the form

$$
\begin{gathered}
\frac{1}{Z_{q}^{(0)}\left(\bar{X} \cup \operatorname{supp} \mathscr{C} \mid \phi_{\partial \Lambda^{c}}\right)} e^{c L^{-v}|P|} e^{-\left(\tau / 3-c L^{-v}-f_{1}\left(1+B^{2}\right)\right)|\operatorname{supp} \mathscr{C}|} \\
\cdot e^{\left(c L^{-v}+f_{1}\left(1+\dot{B}^{2}\right)\right)|\bar{X}|} \int d \phi_{\bar{X}} \tilde{F}_{X}\left(\phi_{X}\right) e^{-H_{q}^{(0)}\left(\phi_{\bar{X}} \mid \phi_{\left.\partial \Lambda^{c}\right)}\right.} e^{(\varepsilon / 2)} \sum_{x \in \partial X(\mathscr{Q})} \phi_{x}^{2}
\end{gathered}
$$

Bounding the integral by

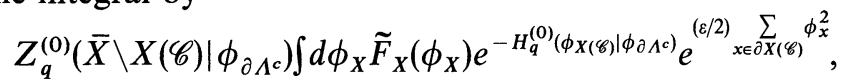

and the ratio

$$
\frac{Z_{q}^{(0)}\left(\bar{X} \backslash X(\mathscr{C}) \mid \phi_{\partial \Lambda^{c}}\right)}{Z_{q}^{(0)}\left(\bar{X} \cup \operatorname{supp} \mathscr{C} \mid \phi_{\partial \Lambda^{c}}\right)} \leqq e^{\tilde{f}_{2}\left(1+B^{2}\right)|X(\mathscr{C}) \cup \operatorname{supp} \mathscr{C}|}
$$

with a constant $\tilde{f}_{2}$ depending only on $v, \eta, \eta^{\prime}$ and $\eta^{\prime \prime}$, we continue as in [1] to complete the proof of Proposition 4.1.

At this point the proof of Lemma 2.5 is a standard exercise: one repeats the procedure leading to the polymer representation (4.7) for the unnormalized expectation value $[A]_{\Lambda, R_{\partial \Lambda^{c}}}^{q}=\langle A\rangle_{\Lambda, R_{\partial \Lambda^{c}}}^{q} Z_{q}\left(\Lambda \mid \phi_{\partial \Lambda^{c}}\right)$ and uses the usual algebraic procedure to divide out the partition function $Z_{q}\left(\Lambda \mid \phi_{\partial \Lambda^{c}}\right)$. Since we have assumed that the observable $A$ is bounded, the bound proven in Proposition 4.1 immediately gives the bounds necessary to prove the convergence of the resulting cluster expansion for $\langle A\rangle_{z, R_{\partial \Lambda}}^{q}$. Lemma 2.5 is an immediate consequence.

\section{Periodic Boundary Conditions}

In this section we analyse periodic boundary conditions. We first consider the partition function,

$$
Z_{\text {per }}(T)=\int d R_{T} e^{-H\left(R_{T}\right)},
$$

where $T$ is the $v$-dimensional torus with length $l$ in each direction. As usual we assume that $T$ is a union of $L$-blocks, i.e. $l$ is an integer multiple of $L$, where $L$ is chosen according to Lemma 3.1 in [1]. Denoting by $h_{q}^{\prime}$ the free energy of the truncated contour model (see Sect. 3 of [1] for the precise definition), by $h$ the minimum of the $h_{q}^{\prime}$ 's and by $a_{q}$ the difference $h_{q}^{\prime}-h$, we recall that the phase $q$ is 
stable iff $a_{q}=0$ and introduce the constant

$$
\tilde{a}=\min _{m: a_{m} \neq 0} a_{m} .
$$

Our first goal is the proof of the following theorem, which is the appropriate reformulation of the corresponding theorem in [9]. $\mathscr{V}_{1}$ is the neighborhood of $0 \in \mathbf{R}^{N-1}$ defined in Lemma 3.1 of [1].

Theorem 5.1. Assume that $V$ and $W$ obey the conditions A.0 through A.3 of [1]. Then there are constants $\beta^{*}<\infty, B>0$ and $l_{0}=l_{0}(\tilde{a})<\infty$, such that for $\mu \in \mathscr{V}_{1}$, $\beta \geqq \beta^{*}$ and $l \geqq l_{0}$,

$$
\left|Z_{\text {per }}(T) e^{h l^{v}}-N_{0}\right| \leqq e^{-B l}
$$

$N_{0}$ is the number of stable phases.

The proof of Theorem 5.1 is essentially the same as that of the corresponding theorem in [9]. For the convenience of the reader and for later reference, however, we give the complete proof. We start with the partition of unity,

$$
1=\sum_{\omega: T \rightarrow\{0,1, \ldots, N\}} \prod_{x \in T} \chi_{\omega(x)}\left(R_{x}\right)
$$

with $\chi_{\omega}(\cdot)$ as before and define a $L$-block, $\square\left(x^{\prime}\right)$, to be $m$-correct iff $\omega(x)=m$ for all $x$ with dist $\left(x, \square\left(x^{\prime}\right)\right) \leqq 1$. As before the connected components of the set, $B(\omega)$, of incorrect points are the supports, supp $\gamma_{1}, \ldots, \operatorname{supp} \gamma_{n}$, of the contours, $\gamma_{1}=\left(\operatorname{supp} \gamma_{1}, \omega_{\gamma_{1}}\right), \ldots, \gamma_{n}=\left(\operatorname{supp} \gamma_{n}, \omega_{\gamma_{n}}\right)$, of the configuration $\omega$.

Following [9] we first consider those configurations, $\omega$, for which

$$
\operatorname{diam} \gamma \leqq l / 3 \text { for all contours } \gamma \text {. }
$$

For such configurations, the notation of exterior contours can be well defined: For a contour, $\gamma$, of $\omega$ let Int $\gamma$ be the union of those components of $T \backslash \operatorname{supp} \gamma$ which have diameter less or equal $l / 3$. Ext $\gamma$ is then the remaining component of $T \backslash \operatorname{supp} \gamma$, and the set of exterior contours of the configuration $\omega$ is the set of contours, $\gamma$, for which there is no other contour, $\gamma^{\prime}$, of $\omega$ such that supp $\gamma \subset \operatorname{Int} \gamma^{\prime}$. Denoting the sum over all configurations, $\omega$, which obey (5.4) and whose exterior contours are $m$-contours by $\sum^{(m)}$, we introduce the partition function

$$
\tilde{Z}_{m}(T)=\sum_{\omega}^{(m)} \int \prod_{x \in T} \chi_{\omega(x)}\left(R_{x}\right) e^{-H\left(R_{T}\right)} d R_{T}
$$

More generally, we define, for a subset $V \subset T$,

$$
\tilde{Z}_{m}(V)=\sum_{\omega}^{(m)} \int \prod_{x \in V} \chi_{\omega(x)}\left(R_{x}\right) e^{-H\left(R_{V}\right)} d R_{V}
$$

where we impose the additional constraint that all exterior contours, $\gamma$, obey the "diluteness condition" $\operatorname{dist}(V(\gamma), T \backslash V) \geqq L$. As before $V(\gamma)$ is the set Int $\gamma \cup \operatorname{supp} \gamma$. We also introduce the partition function,

$$
Z_{\mathrm{Big}}(T)=\sum_{\omega}^{\mathrm{Big}} \int \prod_{x \in T} \chi_{\omega(x)}\left(R_{x}\right) e^{-H\left(R_{T}\right)} d R_{T},
$$

where the sum goes over all configurations violating the condition (5.4). 
With this notation we rewrite $Z_{\text {per }}(T)$ as

$$
Z_{\text {per }}(T)=\sum_{m=1}^{N} \tilde{Z}_{m}(T)+Z_{\text {Big }}(T) .
$$

Theorem 5.1 now follows immediately from (5.7) and the following three lemmas, which are proven in the sequel. We assume from now on that $V$ and $W$ obey assumptions A.0 through A.3 of [1], and that $\mu \in \mathscr{V}_{1}, L \geqq L_{0}$ and $\beta \geqq \beta_{2}$, where $L_{0}$ and $\beta_{2}$ are the constants from Lemma 3.2 and Theorem 3.3 of [1], respectively.

Lemma 5.2. Assume that $q$ is stable. Then there are constants $K_{2}<\infty, B_{2}>0$ such that

$$
\left|\log \tilde{Z}_{q}(T)-h\right| T \mid \leqq K_{2} e^{-B_{2} l} .
$$

Lemma 5.3. There is a constant $\beta_{3}<\infty$, such that for $\beta \geqq \beta_{3}$ the following statements are true:

i) For all $m \in\{1, \ldots, N\}$ and all $V \subset T$ which are unions of L-blocks

$$
\tilde{Z}_{m}(V) e^{h|V|} \leqq \exp \left\{K_{2} e^{-B_{2} l}+\left(C_{1}+1\right)\left|\partial V^{c}\right|\right\} .
$$

ii) If $m$ is unstable

$$
\tilde{Z}_{m}(T) e^{h|T|} \leqq e^{K_{2}} \max \left\{e^{-\left(a_{m} / 2\right) l^{v}}, e^{-B_{3} \tau l l^{\nu-1}}\right\} .
$$

Here $\tau=\tau(\beta, L)$ is the constant from Lemma 3.1 [1] and $B_{3}>0$ is a constant depending only on the dimension.

Lemma 5.4. There is a constant $\beta_{4}<\infty$ such that

$$
Z_{\mathrm{Big}}(T) e^{h|T|} \leqq e^{-\tau l / 6}
$$

provided $\beta \geqq \beta_{4}$.

Proof of Lemma 5.2. For the Gaussian approximation, $Z_{q}^{(0)}(T)$, to $\tilde{Z}_{q}(T)$, the statement is well known. It can be proven, for example, by comparing the random walk expansion for $\log Z_{q}^{(0)}(T)$ with the random walk expansion for $|T| h_{q}^{(0)}$. Since the difference between $\log Z_{q}^{(0)}(T)$ and $\log \tilde{Z}_{q}(T)$ can be controlled by a convergent cluster expansion if $q$ is stable, the result immediately generalizes to $\log \tilde{Z}_{q}(T)$.

Proof of Lemma 5.3. We proceed as in the proof of Theorem 3.3, [1] to bound

$$
\tilde{Z}_{m}(V) e^{h|V|} \leqq \exp \left\{C_{1}\left|\partial V^{c}\right|+K_{2} e^{-B_{2} l}\right\} \sum_{\mathscr{C}}^{\prime} e^{-a_{m} \mid V \backslash \text { Int } \mathscr{C} \mid} \prod_{\gamma \in \mathscr{C}} e^{-\left(\tau-3 C_{1}-1\right)|\operatorname{supp} \gamma|},
$$

where the sum goes over sets $\mathscr{C}$ of mutally external $m$-contours which are all large (i.e. $a_{m} \operatorname{diam} \gamma>\tau / 3$ ) and obey a bound diam $\gamma<l / 3$. Extracting a factor

$$
M=\max _{\mathscr{C}} e^{-\left(a_{m} / 2\right)|V \backslash \operatorname{Int} \mathscr{C}|} \prod_{\gamma \in \mathscr{C}} e^{-(\tau / 2)|\mathscr{C}|}
$$

and continuing as in the proof of Theorem 3.3, [1], we obtain, for a suitable choice of $\beta_{3}$ and for $\beta \geqq \beta_{3}$,

$$
\tilde{Z}_{m}(V) e^{h|V|} \leqq M \exp \left\{\left(C_{1}+1\right)\left|\partial V^{c}\right|+K_{2} e^{-B_{2} l}\right\} .
$$


Bounding $M$ by 1 we obtain i). To prove ii) we set $V=T$ and use the isoperimetric inequality to bound

$$
|\mathscr{C}| \geqq\left|\partial(\operatorname{Int} \mathscr{C})^{c}\right| \geqq K|\operatorname{Int} \mathscr{C}|^{(v-1) / v},
$$

where $K>0$ is a constant depending only on the dimension $v$. We obtain that

$$
M \leqq \sup _{0 \leqq x \leqq|T|} e^{\left(a_{m} / 2\right)(|T|-x)} e^{-(\tau / 2) K x^{(v-1) / v}} .
$$

The supremum is obtained for either $x=0$ or $x=|T|$, which shows that

$$
M \leqq \max \left\{e^{-\left(a_{m} / 2\right)|T|}, e^{-(\tau / 2) K|T|(v-1) / v}\right\} .
$$

This proves ii), with, for example, $B_{3}=K / 2$.

Proof of Lemma 5.4. For a given configuration $\omega$ contributing to (5.6) we let $\mathscr{C}_{1}$ be the union of those contours which violate (5.4) and $V_{m}$ be the union of those components, $K$, of $T \backslash \operatorname{supp} \mathscr{C}_{1}$ for which $\omega=m$ on $\partial K$. Resumming all configurations $\omega$ which give rise to the same set $\mathscr{C}_{1}$ we may bound

$$
Z_{\text {Big }}(T) \leqq \sum_{\mathscr{C}_{1}} \prod_{m} \tilde{Z}_{m}\left(V_{m}\right) \prod_{\gamma \in \mathscr{C}_{1}} e^{-\left(\tau+h-C_{1}\right)|\gamma|},
$$

where we used Lemma 3.1 and Lemma 3.2,ii) of [1] to bound integrals of the form

$$
\int d R_{\gamma} \chi_{\omega_{\gamma}}\left(R_{\gamma}\right) e^{-H\left(R_{\gamma} \mid R_{\partial(\operatorname{supp} \gamma)^{c}}\right)}, \quad \gamma \in \mathscr{C}_{1} .
$$

Next we use Lemma 5.3, i), to conclude that

$$
Z_{\mathrm{Big}}(T) e^{h|T|} \leqq e^{K_{2}} \sum_{\mathscr{C}_{1}} \prod_{\gamma \in \mathscr{C}_{1}} e^{-\left(\tau-2 C_{1}-1\right)|\gamma|} .
$$

Bounding the sum over all contours, $\gamma$, with fixed size $s=|\gamma|$ by $l^{v} e^{K s}$, where $K$ is a constant depending only on $v$ and $N$, we obtain, for $\tau$ large enough,

$$
Z_{\mathrm{Big}}(T) e^{h|T|} \leqq e^{K_{2}} \sum_{n=1}^{\infty} \frac{1}{n !}\left(l^{\nu} \sum_{s \geqq l / 3} e^{-\left(\tau-K-2 C_{1}-1\right) s}\right)^{n} \leqq e^{-\tau l / 6} .
$$

We are now ready to prove Theorem B of Sect. 1. We consider a bounded, local observable, $A$, and introduce the modified partition function,

$$
\tilde{Z}_{m}(A, T)=\sum_{\omega: T \rightarrow\{0,1, \ldots, N\}}^{(m)} \int d R_{T} \prod_{x \in T} \chi_{\omega(x)}\left(R_{x}\right) A e^{-H\left(R_{T}\right)},
$$

as well as

$$
Z_{\text {Big }}(A, T)=\sum_{\omega: T\{0,1, \ldots, N\}}^{\prime} \int d R_{T} \prod_{x \in T} \chi_{\omega(x)}\left(R_{x}\right) A e^{-H\left(R_{T}\right)},
$$

where the sum $\sum^{\prime}$ denotes a sum over all configurations, $\omega$, which violate the condition (5.4). We also introduce the expectation values

$$
\langle A\rangle_{m, T}^{\prime}=\frac{\tilde{Z}_{m}(A, T)}{\tilde{Z}_{m}(T)}
$$

and recall that $\langle A\rangle_{q}$ denotes the infinite volume limit of the finite volume expectation values with boundary condition $q$, see [1], Theorem 1. For stable 
values of $q$, the difference, $\langle A\rangle_{q}-\langle A\rangle_{m, T}^{\prime}$, can be controlled by a convergent cluster expansion and goes to zero as $T \rightarrow \mathbf{Z}^{v}$. Therefore Theorem B follows immediately from the following theorem.

Theorem 5.5. Assume that $V$ and $W$ obey the assumptions A.0 through A.3 of [1]. Then there are constants $\beta^{*}<\infty, B>0$ and $l_{0}=l_{0}(\tilde{a})$, such that

$$
\left|\langle A\rangle_{\mathrm{per}, T}-\frac{1}{N_{0}} \sum_{q}\langle A\rangle_{q, T}^{\prime}\right| \leqq\|A\| e^{-B l},
$$

provided $\mu \in \mathscr{V}_{1}, \beta \geqq \beta^{*}$ and $l \geqq l_{0}$. The sum goes over all stable values of $q$, and $N_{0}$ denotes the number of stable phases.

Proof. Theorem 5.5 immediately follows from the identity

$$
\langle A\rangle_{\mathrm{per}, T}=\frac{1}{Z_{\mathrm{per}}(T)}\left[\sum_{m=1}^{N} \tilde{Z}_{m}(A, T)+Z_{\mathrm{Big}}(A, T)\right],
$$

Theorem 5.1, Lemma 5.2 through 5.4 and the bounds

$$
\begin{aligned}
\tilde{Z}_{m}(A, T) & \leqq\|A\| \tilde{Z}_{m}(T), \\
Z_{\mathrm{Big}}(A, T) & \leqq\|A\| Z_{\mathrm{Big}}(T) .
\end{aligned}
$$

Remark. For an unbounded observable $A$, the bound (5.12) is useless because $\|A\|=\infty$. To prove Theorem $\mathrm{B}$ for the more general class of observables for which $\|A\|^{(\alpha)}<\infty$, see [1], Sect. 1, for the definition of $\|A\|^{(\alpha)}$, we show that

$$
\begin{aligned}
& \tilde{Z}_{m}(A, V) e^{h|V|} \leqq\|A\|^{(\alpha)} K^{|\operatorname{supp} A|} \exp \left\{K_{2} e^{-B_{2} l}+\left(C_{1}+1\right)\left|\partial V^{c}\right|\right\}, \\
& \tilde{Z}_{m}(A, T) e^{h|T|} \leqq\|A\|^{(\alpha)} K^{|\operatorname{supp} A|} e^{K_{2}} \max \left\{e^{-\left(a_{m} / 2\right) l^{v}}, e^{-B_{3} l^{v-1}}\right\}
\end{aligned}
$$

and

$$
Z_{\text {Big }}(A, T) e^{h|T|} \leqq\|A\|^{(\alpha)} K^{|\operatorname{supp} A|} e^{-\tau l / 6},
$$

with a constant $K$ which does not depend on $l$. Given these bounds we immediately obtain Theorem 5.5 with $\|A\|$ replaced by $\|A\|^{(\alpha)} K^{|\operatorname{supp} A|}$, and hence Theorem $\mathrm{B}$ for all observables, $A$, with $\|A\|^{(\alpha)}<\infty$. Since the proof of (5.15) to (5.17) is a straightforward combination of the methods developed in Sect. 7 of [1] and the methods of this section, we don't present the details here.

\section{Appendix: Sswerstability Estimates}

In this appendix we prove Lemma 2.3 , using the methods of [2-4]. In fact we prove a more general theorem on the probability that $\alpha\left(R_{x}\right) \geqq \alpha_{0}$ for a given site, $s \in \mathbf{Z}^{v}$. Lemma 2.3 then follows by a three line argument. To make this appendix self-contained we restate those assumptions which are needed in the course of the proof.

We consider a spin model with spins $R_{x} \in \mathbf{R}$ and a Hamiltonian

$$
H=\sum_{x} V\left(R_{x}\right)+\sum_{\langle x y\rangle} W\left(R_{x}, R_{y}\right)
$$


where the sum $\sum_{\langle x y\rangle}$ denotes a sum over nearest neighbor pairs, $\langle x y\rangle$. We assume that $V$ and $W$ are bounded from below and that $W$ is normalized in such a way that $W\left(R_{x}, R_{y}\right) \geqq 0$. Putting

$$
\alpha\left(R_{x}\right)=V\left(R_{x}\right)-\inf _{R \in \mathbf{R}} V(R)
$$

we assume that $W$ obeys the regularity condition

and that

$$
0 \leqq W\left(R_{x}, R_{y}\right) \leqq k_{1}\left(1+\alpha\left(R_{x}\right)+\alpha\left(R_{y}\right)\right)
$$

$$
\int e^{-(1-\lambda) \alpha(R)} d R<\infty
$$

for some constants $k_{1}<\infty, 0<\lambda<1$. For the model considered in the main body of this paper assumption (A.2) is the assumption $R$ of Sect. 1 and (A.3) immediately follows from assumptions A.1 i) and A.2 ii) of [1].

Let $\Lambda(j)$ be the set of all points in $\mathbf{Z}^{v}$ which have distance ${ }^{10}$ less or equal to $j$ from the origin and put, for a configuration $\underline{R}$ in $\mathbf{Z}^{v}$

$$
\|\underline{R}\|=\sup _{j \geqq 0} \frac{1}{\mid \Lambda(j)} \sum_{x \in \Lambda(j)} \alpha\left(R_{x}\right) .
$$

We define: an equilibrium state, $\langle\cdot\rangle=\int \cdot d P$, is tempered if $\|\underline{R}\|<\infty$ with probability one.

The goal of this appendix is to prove the following:

Theorem A.1. Let $\langle\cdot\rangle=\int \cdot d P$ be a tempered equilibrium state. Then there is a constant $K<\infty$ such that the probability, $P\left(\alpha\left(R_{x}\right)>\alpha_{0}\right)$, that $\alpha\left(R_{x}\right)$ is larger than $\alpha_{0}$ is bounded from above by $\mathrm{Ke}^{-(\lambda / 2) \alpha_{0}}$.

Remarks

i) As the reader may easily verify from the following proof, the constant $\lambda / 2$ in the above theorem may be replaced by any constant $\tilde{\lambda}<\lambda$, at the cost of making $K$ larger if $\lambda / 2<\tilde{\lambda}$.

ii) Lemma 2.3 immediately follows from Theorem A.1 because, for $a>2(v-1) / \lambda$, the probability that $\alpha\left(R_{x}\right)>a \log j$ is bounded by

$$
K\left(\frac{1}{j}\right)^{\lambda a / 2} \leqq o\left(\left(\frac{1}{j}\right)^{v-1}\right) .
$$

Therefore the probability that $\alpha\left(R_{x}\right)>a \log j$ for at least one $x$ with $\operatorname{dist}(x, 0)=j$ is bounded by

$$
O\left(j^{v-1}\right) o\left(\left(\frac{1}{j}\right)^{v-1}\right)
$$

which goes to zero as $j \rightarrow \infty$.

\footnotetext{
${ }^{10}$ We define the distance dist $(x, y)$ between two points, $x, y \in \mathbf{Z}^{v}$, as $|x-y|_{1}$, where $|\cdot|_{1}$ denotes the $l_{1}$ distance in $\mathbf{Z}^{v}$
} 
Proof of Theorem A.1. We introduce a growing sequence of positive numbers $\psi_{k} \rightarrow \infty$ as $k \rightarrow \infty$ and a sequence of volumes $\Lambda_{k}$, such that

i) $\Lambda_{0}$ contains all $y \in \mathbf{Z}^{v}$ with $\operatorname{dist}(x, y) \leqq 1$,

ii) $\partial \Lambda_{k}^{c} \subset \Lambda_{k+1}$ and

iii) $\frac{\left|\Lambda_{k+1}\right|}{\left|\Lambda_{k}\right|} \frac{\psi_{k+1}}{\psi_{k}} \leqq 1+\varepsilon$

for some small number $\varepsilon$ to be chosen later (a possible choice would be, for example, $\Lambda_{k}=\Lambda\left(k+k_{0}\right), \psi_{k}=k+k_{0}$, where $k_{0}$ has been chosen large enough, depending on ع). As in [2-4] we define sets, $\mathscr{C}_{k}$, of configurations $\underline{R}$,

$$
\begin{aligned}
\mathscr{C}_{0} & =\left\{\underline{R}\left|\sum_{x \in \Lambda_{k}} \alpha\left(R_{x}\right)<\psi_{k}\right| \Lambda_{k} \mid \forall k \geqq 0\right\} \\
\mathscr{C}_{j+1} & =\left\{\underline{R}\left|\sum_{x \in \Lambda_{j}} \alpha\left(R_{x}\right) \geqq \psi_{j}\right| \Lambda_{j}\left|, \sum_{x \in \Lambda_{k}} \alpha\left(R_{x}\right)<\psi_{k}\right| \Lambda_{k} \mid \forall k \geqq j+1\right\} .
\end{aligned}
$$

We also introduce the characteristic functions, $\chi_{j}=\chi_{j}(\underline{R})$, which are 1 if $\underline{R} \in \mathscr{C}_{j}$ and 0 otherwise. Since $P$ is tempered, we may rewrite

$$
P\left(\alpha\left(R_{x}\right)>\alpha_{0}\right)=\sum_{j=0}^{\infty} P_{j}
$$

with

$$
P_{j}=\int d P(\underline{R}) \chi_{j}(\underline{R}) \chi\left(\alpha\left(R_{x}\right)>\alpha_{0}\right) .
$$

Using the DLR equations, we rewrite

$$
P_{j+1}=\int d P\left(R_{\Lambda_{j}^{c}}\right) \frac{1}{Z\left(\Lambda_{j} \mid R_{\partial \Lambda_{j}^{c}}\right)} \int d R_{\Lambda_{j}} \chi\left(\alpha\left(R_{x}\right)>\alpha_{0}\right) \chi_{j+1}(\underline{R}) e^{-H\left(R_{\Lambda_{j}} \mid R_{\Lambda_{j}^{c}}\right)}
$$

where we used the convention $\Lambda_{-1}=\{x\}$.

After these preparations we bound the probability $P\left(\alpha\left(R_{x}\right)>\alpha_{0}\right)$. We first estimate $P_{0}$. The necessary lower bound on $Z\left(\{x\} \mid R_{\partial\{x\} c}\right)=\int d \tilde{R}_{x} e^{-H\left(\tilde{R}_{x} \mid R_{\partial(x) c}\right)}$ is obtained as follows. For $\underline{R} \in \mathscr{C}_{0}$ and $\tilde{R}_{x} \in \mathbf{R}$ we estimate

$$
\begin{aligned}
H\left(\tilde{R}_{x} \mid R_{\partial\{x\}^{c}}\right) & \leqq V\left(\tilde{R}_{x}\right)+k_{1} \sum_{y:|y-x|=1}\left(1+\alpha\left(\tilde{R}_{x}\right)+\alpha\left(R_{y}\right)\right) \\
& \leqq V\left(\tilde{R}_{x}\right)+2 v k_{1}\left(1+\alpha\left(\tilde{R}_{x}\right)\right)+k_{1} \sum_{y \in \Lambda_{0}} \alpha\left(R_{y}\right) \\
& \leqq V\left(\tilde{R}_{x}\right)+2 v k_{1}\left(1+\alpha\left(\tilde{R}_{x}\right)\right)+k_{1} \psi_{0}\left|\Lambda_{0}\right|
\end{aligned}
$$

which, for $\underline{R} \in \mathscr{C}_{0}$, gives the bound

$$
\frac{1}{Z\left(\{x\} \mid R_{\partial\{x\}^{c}}\right)} \leqq e^{k_{1} \psi_{0}\left|\Lambda_{0}\right|} C
$$

with $C=e^{2 v k_{1}} / \int d \tilde{R} e^{-V(\tilde{R})-2 v k_{1} \alpha(\tilde{R})}$. Inserting this bound into (A.10) we obtain, using 
the lower bound $W\left(R, R^{\prime}\right) \geqq 0$,

$$
P_{0} \leqq e^{k_{1} \psi_{0}\left|\Lambda_{0}\right|} C \int_{\alpha\left(R_{x}\right) \geqq \alpha_{0}} e^{-V\left(R_{x}\right)} d R_{x} \leqq e^{k_{1} \psi_{0}\left|\Lambda_{0}\right|} \tilde{C} e^{-\lambda \alpha_{0}},
$$

with

$$
\tilde{C}=C \int e^{\lambda \alpha(R)-V(R)} d R=e^{2 v k_{1}} \frac{\int e^{-V(R)+\lambda \alpha(R)} d R}{\int e^{-V(\tilde{R})-2 v k_{1} \alpha(\tilde{R})} d \tilde{R}} .
$$

To bound $P_{j+1}$ for $j \geqq 0$ we estimate, for $\underline{R} \in \mathscr{C}_{j+1}$ and $\tilde{R}_{\Lambda_{j}}: \Lambda_{j} \rightarrow \mathbf{R}$,

$$
\begin{aligned}
H\left(\tilde{R}_{\Lambda_{j}} \mid R_{\partial \Lambda_{j}^{c}}\right) & \leqq \sum_{x \in \Lambda_{j}} V\left(\tilde{R}_{x}\right)+\sum_{\substack{x \in \Lambda_{j}, y \in \partial \Lambda_{j}^{c} \\
|x-y|=1}}\left(1+\alpha\left(\tilde{R}_{x}\right)+\alpha\left(R_{y}\right)\right) \\
& \leqq \sum_{x \in \Lambda_{j}}\left\{V\left(\tilde{R}_{x}\right)+2 v k_{1}\left(1+\alpha\left(\tilde{R}_{x}\right)\right)\right\}+2 v k_{1} \sum_{y \in \Lambda_{j+1} \backslash \Lambda_{j}} \alpha\left(R_{y}\right) \\
& \leqq \sum_{x \in \Lambda_{j}}\left\{V\left(\tilde{R}_{x}\right)+2 v k_{1}\left(1+\alpha\left(\tilde{R}_{x}\right)\right)\right\}+2 v k_{1}\left(\frac{\left|\Lambda_{j+1}\right|}{\left|\Lambda_{j}\right|} \frac{\psi_{j+1}}{\psi_{j}}-1\right) \sum_{x \in \Lambda^{j}} \alpha\left(R_{x}\right),
\end{aligned}
$$

where we have used (A.6) in the second step. Choosing the constant $\varepsilon$ in (A.7) so small that $\left(2 v k_{1}\right) \varepsilon \leqq \lambda / 4$ we obtain the bound

$$
\begin{aligned}
\frac{1}{Z\left(\Lambda_{j} \mid R_{\partial \Lambda_{j}^{c}}\right)} & \leqq C^{\left|\Lambda_{j}\right|} e^{-(\lambda / 4) \sum_{y \in \Lambda_{j}} \alpha\left(R_{y}\right)} \\
& \leqq e^{-(\lambda / 2) \alpha_{0}}\left(C e^{\left.-(\lambda / 4) \psi_{j}\right)^{\left|\Lambda_{j}\right|}} e^{\lambda \sum_{y \in \lambda_{j}} \alpha\left(R_{y}\right)}\right.
\end{aligned}
$$

provided $\underline{R} \in \mathscr{C}_{j+1}$ and $\alpha\left(R_{x}\right) \geqq \alpha_{0}$ (we have used the fact that $x \in \Lambda_{j}$ for all $j \geqq 0$ by our choice of $\Lambda_{j}$, see (A.5) and A.6)). Inserting the above bound into (A.10) one gets the estimate

$$
P_{j+1} \leqq e^{-(\lambda / 2) \alpha_{0}}\left(\tilde{C} e^{-(\lambda / 4) \psi_{j}}\right)^{\left|\Lambda_{j}\right|} .
$$

Combined with (A.11) we finally obtain Theorem A.1 with

$$
K=\tilde{C} e^{k_{1} \psi_{0}\left|\Lambda_{0}\right|}+\sum_{j \geqq 0}\left(\tilde{C} e^{-(\lambda / 4) \psi_{j}}\right)^{\left|\Lambda_{\jmath}\right|} .
$$

\section{References}

1. Borgs, C., Waxler, R.: First order phase transitions in unbounded spin systems. I. Construction of the Phase Diagram, Commun. Math. Phys. 126, 291-324 (1989)

2. Ruelle, D.: Superstable interactions in classical statistical mechanics. Commun. Math. Phys. 18, 127 (1970)

3. Ruelle, D.: Probability estimates for continuous spin systems. Commun. Math. Phys. 50, 189(1976)

4. Lebowitz, J. L., Presutti, E.: Statistical mechanics of systems of unbounded spins. Commun. Math. Phys. 50, 195 (1976)

5. Gallavotti, G., Martin-Löf, A., Miracle-Solé, S.: Some problems connected with the description of coexisting phases at low temperatures in the Ising model. In: Statistical mechanics and mathematical problems (Batelle, Seattle, 1971), A. Lenard (ed). Lecture Notes in Phys., vol. 20. Berlin, Heidelberg, New York: Springer 1973 
6. Dobrushin, R. L.: The description of a random field by means of conditional probabilities and conditions on its regularity. Theor. Prob. Appl. 13, 197 (1968)

7. Lanford, O., Ruelle, D.: Observables at infinity and states with short range correlations in statistical mechanics. Commun. Math. Phys. 13, 194 (1969)

8. Zahradnik, M.: An alternative version of Pirogov-Sinai theory. Commun. Math. Phys. 93, 559 (1984)

9. Borgs, C., Imbire, J.: A unified approach to phase diagrams in field theory and statistical mechanics. Commun. Math. Phys. 123, 305-328 (1989)

Communicated by J. Fröhlich

Received February 24, 1989 